\title{
Custom Wide Counterflow Pipelines for High-Performance Embedded Applications
}

\author{
Bruce R. Childers, Member, IEEE, and Jack W. Davidson, Member, IEEE
}

\begin{abstract}
Application-specific instruction set processor (ASIP) design is a promising technique to meet the performance and cost goals of high-performance systems. ASIPs are especially valuable for embedded computing applications (e.g., digital cameras, color printers, cellular phones, etc.) where a small increase in performance and decrease in cost can have a large impact on a product's viability. Sutherland, Sproull, and Molnar originally proposed a processor organization called the counterflow pipeline (CFP) as a general-purpose architecture. We observed that the CFP is appropriate for ASIP design due to its simple and regular structure, local control and communication, and high degree of modularity. This paper describes a new CFP architecture, called the wide counterflow pipeline (WCFP), that extends the original proposal to be better suited for custom embedded instruction-level parallel processors. This work presents a novel and practical application of the CFP to automatic and quick turnaround design of ASIPs. The paper introduces the WCFP architecture and describes several microarchitecture capabilities needed to get good performance from custom WCFPs. We demonstrate that custom WCFPs have performance that is up to four times better than that of ASIPs based on the CFP. Using an analytic cost model, we show that custom WCFPs do not unduly increase the cost of the original counterflow pipeline architecture, yet they retain the simplicity of the CFP. We also compare custom WCFPs to custom VLIW architectures and demonstrate that the WCFP is performance competitive with traditional VLIWs without requiring complicated global interconnection of functional devices.
\end{abstract}

Index Terms-Counterflow pipelines, application-specific processors, automatic architectural synthesis.

\section{INTRODUCTION}

A PPLICATION-SPECIFIC instruction set processor design (ASIP) is a promising approach for improving the cost-performance ratio of an application. ASIPs are especially useful for embedded applications (e.g., digital cameras, cellular phones, color printers, etc.) where a small increase in performance and decrease in cost can have a large impact on a product's viability. A novel computer organization, called the counterflow pipeline (CFP) [1], has several characteristics that make it attractive for custom embedded processors. The CFP has a simple and regular structure, local control, high degree of modularity, synchronous and asynchronous implementations, and inherent handling of complex features such as register renaming and pipeline interlocking. However, the CFP is not performance competitive for ASIPs. In this paper, we extend the CFP to a wide-issue organization, called a wide counterflow pipeline (WCFP), that has much higher performance and is better suited for automatic design of instruction-level parallel (ILP) processors than the original proposal. Our work is a novel and practical application of the CFP to automatic ASIP design.

General-purpose processors are good for average workloads, as typified by popular benchmark suites such as SPEC CPU2000 [2]. These architectures are optimized to

- B.R. Childers is with the Department of Computer Science, University of Pittsburgh, 210 S. Bouquet St., Pittsburgh, PA 15260.

E-mail: childers@cs.pitt.edu.

- J.W. Davidson is with the Department of Computer Science, University of Virginia, 151 Engineer's Way, Charlottesville, VA 22904.

E-mail: jwd@cs.virginia.edu.

Manuscript received 12 Nov. 2001; revised 14 Jan. 2003; accepted 14 Jan. 2003.

For information on obtaining reprints of this article, please send e-mail to: tc@computer.org, and reference IEEECS Log Number 115356. execute applications containing a set of common operations and exhibiting similar behavior. The ever increasing demand for performance and the need to handle arbitrary code has led to very complex and costly general-purpose microarchitectures. Although the cost of aggressive generalpurpose processors is usually prohibitive for embedded applications, many embedded applications would benefit from high performance. An alternative to a general-purpose architecture is a custom processor matched to an application's performance and cost goals. An ASIP has the flexibility to include the minimal instruction set and microarchitecture elements that give good performance and low cost (e.g., power consumption, code size, and quick turn-around design) for a single code without the complexity of devices for general-purpose codes. Because cost and time-to-market are very important to embedded systems [3], an ASIP architecture should permit automatic design, including high-level architectural design.

The WCFP is a good candidate for this type of fast architectural synthesis because of its superior composability and simplicity. This greatly reduces the complexity of automatic design because a synthesis system does not have to design control paths and determine complex bus and bypass networks as it would for a traditional architecture, such as a custom VLIW [4]. The WCFP also has local pointto-point communication between functional blocks, which may lead to faster and lower power processors than architectures that use traditional structures for global interconnection of functional devices. Local communication is especially important as feature size is reduced, where wire latency and power consumption dominates transistor latency and power [5].

While our previous work used the CFP as a target for application-specific processors [6] [7], this paper describes a 
new wide-issue counterflow pipeline architecture. This new architecture is better suited for custom ILP processors than the CFP. Although ASIPs based on the CFP take some advantage of ILP, they do not fully exploit an application's ILP because they issue only one instruction at a time. We observed that higher levels of performance may be obtained by widening the CFP's instruction pipeline to take better advantage of ILP with the assistance of compiler transformations such as if-conversion and software pipelining.

The WCFP is ideal for automatic design of ILP processors because its pipeline width, depth, and functional repertoire can be easily and quickly tailored to match the performance requirements of an application. Indeed, we have developed an end-to-end architectural design system that accepts an application in the programming language $C$ and automatically generates a custom processor for that application using the WCFP. Our previous work describes the design methodology [8] and software infrastructure [9] needed for architectural synthesis of custom WCFPs. This paper presents the WCFP architecture and several microarchitecture capabilities needed to get high performance, including result packing, register caching, and predicated execution. We also develop a cost model for evaluating custom CFPs and WCFPs. This model can be used to investigate the costs associated with different alternatives when constructing custom WCFPs. We use the model to explore the relative cost difference in WCFP implementations for several benchmarks. We also evaluate WCFP cost versus the CFP. Finally, we demonstrate that WCFPs with local communication between functional blocks are performance competitive with traditional custom VLIW architectures.

This paper is organized as follows: Section 2 describes our target applications, general system architecture, and background material about the original counterflow pipeline. Section 3 presents the WCFP and several microarchitecture capabilities needed to get good performance from custom WCFPs. Section 4 has a performance evaluation of the WCFP and its microarchitecture features. We also compare the WCFP to ASIPs based on traditional customization methodologies using VLIWs. Section 5 describes a cost model for evaluating WCFP design alternatives. Section 6 presents related work and Section 7 summarizes the paper.

\section{BACKGROUND}

In this section, we first describe our target applications and system architecture. We also explain how the CFP [1] works. Finally, we discuss some characteristics of the counterflow pipeline.

\subsection{Design Strategy}

Target Applications. The kinds of high-performance embedded applications that we are targeting with custom WCFPs have two parts: control code and a computationally intensive part. The computation part is a kernel loop that accounts for the majority of execution time. Some applications with this organization include digital cameras (JPEG), color printers (Floyd-Steinberg dithering), and digital video camcorders (MPEG). Increasing the performance of the most frequently executed portion of an application in- creases overall performance. Thus, synthesizing custom hardware for the computation-intensive portion of an application is an effective way to increase performance.

The type of applications we are targeting need only a modest kernel speedup to improve overall performance. For example, JPEG has a function j_rev_dct () that accounts for 60 percent of total execution time. This function consists of applying a single loop twice (to do the inverse discrete cosine transformation), so it is a good candidate for a custom counterflow pipeline. For JPEG, a small kernel speedup of 6 or 7 achieves most of the overall speedup. Many embedded applications we have examined have execution profiles similar to JPEG with one kernel that consists of over 50 percent of the overall execution of the application. We profiled benchmarks from MediaBench [10] and found that most of these applications had one loop that accounted for most of the execution time. These benchmarks included GSM 6.10 speech coding, ADPCM coding, image compression and decompression, MPEG-III audio playback (not in MediaBench), and CCITT G.721 voice coding. In these benchmarks, one kernel loop accounts for 53 percent to 85 percent of execution time. Some of the MediaBench benchmarks, such as osdemo, texgen, epic_dec, pegwit_enc, and pegwit_dec, did not have a single loop that dominated execution time. Here, generating custom hardware for a single loop is not an effective way to improve overall performance.

The question of how to partition an application into its computationally expensive and control parts can be addressed by software/hardware codesign methodologies. In our system, annotations are added to the application source code to identify the computational kernel. The system extracts this kernel and uses it derive an ASIP. For this paper, we used a profile guided partitioning approach to identify the computational portions of benchmark codes.

System Architecture. Our target system architecture has two processors: a traditional processor (e.g., a MIPS core) for executing control code and a WCFP for executing the computation portions of an application. We incorporate a WCFP in the whole system as a loosely coupled coprocessor in a similar manner as other application-specific systems such as RaPiD [11] and PipeRench [12]. We expect the processors to be integrated on the same chip to reduce overhead and system cost.

\subsection{Counterflow Pipelines}

The CFP was originally proposed as a general-purpose single issue architecture [1]. It has two pipelines flowing in opposite directions, as shown in Fig. 1a. One is the instruction pipeline, which carries instructions from a fetch stage (fetch) to a register file stage (regfile). When an instruction issues, an instruction bundle is formed that flows through the pipeline. Fig. $1 \mathrm{~b}$ shows an instruction bundle that holds the instruction opcode $(O P)$, source register names $\left(S N_{1}\right.$ and $\left.S N_{2}\right)$, a destination register name $\left(D N_{1}\right)$, source operand values $\left(S V_{1}\right.$ and $\left.S V_{2}\right)$, and destination value $\left(D V_{1}\right)$. An instruction bundle also has status flags $(F)$ that indicate whether the instruction has executed.

The other pipeline is the results pipeline that carries results from the register file to the instruction fetch stage. Whenever a value is inserted in the result pipeline, a result 


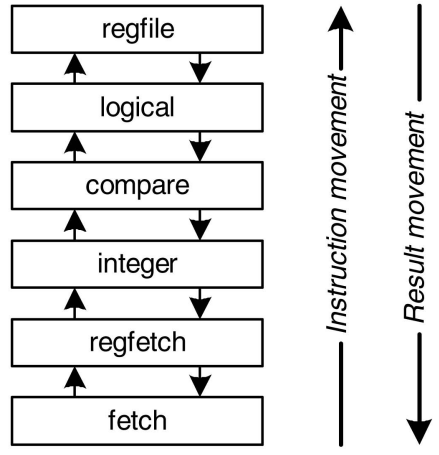

(a)

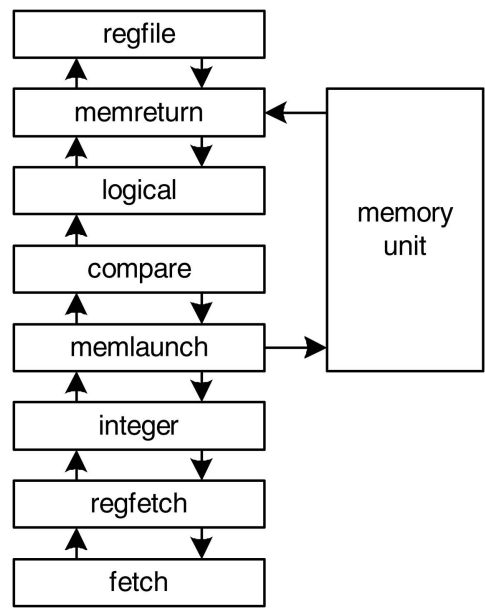

(c)

Instruction Bundle
\begin{tabular}{|c|c|c|c|c|c|c|c|}
\hline $\mathrm{OP}$ & $\mathrm{F}$ & $\mathrm{SN}_{1}$ & $\mathrm{SV}_{1}$ & $\mathrm{SN}_{2}$ & $\mathrm{SV}_{2}$ & $\mathrm{DN}_{1}$ & $\mathrm{DV}_{1}$ \\
\hline
\end{tabular}

(b)

Fig. 1. Example counterflow pipelines and instruction and result bundles. (a) CFP with execute stages. (b) Result and instruction bundles. (c) CFP with functional sidings.

bundle is created that holds the name of a register and its corresponding value. An example result bundle with two registers is shown in Fig. 1b. This result bundle has two register names $\left(R N_{1}\right.$ and $\left.R N_{2}\right)$ and two corresponding values $\left(R V_{1}\right.$ and $\left.R V_{2}\right)$.

A CFP has a series of stages, including instruction fetch, register fetch, execution, and register file writeback. The instruction stage decodes and issues instructions and creates instruction bundles. It also discards results from the pipeline. The register file holds destination register values of instructions that have exited the pipeline. It is updated with an instruction's destination register whenever an instruction enters the stage. The register fetch stage is responsible for fetching register values from the register file stage. Whenever a newly fetched instruction enters the register fetch stage, a request is sent to the register file for the source operands for the new instruction. The source operands are inserted into the result pipeline in a result bundle. Finally, execution stages carry out the actual operations required by instructions. In Fig. 1a, an AND instruction executes in the logical stage, an ADD in the integer stage, and a COMPARE in the compare stage.

The instruction and result pipelines interact: instructions copy values to and from the result pipeline through garnering, killing, or updating. This interaction is governed by rules that ensure sequential execution semantics. There are also rules that ensure result values are current for their position in the pipeline and not values from previous operations that use the same register names.

We illustrate how garner, kill, and update work through an example in Fig. 2. The figure shows the movement and interaction of instructions and results over nine time steps. To simplify the example, assume unit latency; in practice, CFPs have varying latencies for different operations in a stage. Fig. 2a shows a timing diagram of how instructions and results interact, Fig. $2 \mathrm{~b}$ shows the instruction sequence executed by the pipeline, and Fig. 2c shows the result bundles injected into the pipeline. Starting with time 1, instructions I1 and I2 have been fetched and inserted in the pipeline. I1 requests its source operands from the register file. The register file inserts a result bundling holding these sources. On time 2, I1 and I2 advance one stage and a new instruction, I3, is fetched. At time 3, I1 and R1 interact because R1 is carrying the source values needed by I1. A comparison is made with the source names from I1 and with the result register names from $\mathrm{R} 1$. The names match and a garner is done to copy the values of r11 and r12 from R1 to I1. Operand values are copied to an instruction because an instruction can meet its source operand values before reaching an execution stage (e.g., I1 meets its sources in compare and executes in logical).

At time 4, I1 advances to the logical stage, where it can execute, and meets result bundle R2 (requested by I2). A check is made against the names in I1 and R2 and a match is found on I1's destination register name. A kill operation is done to nullify the value of register 10, which will be produced by I1. Killing the value ensures that I2 will not acquire an old value for register 10. A kill is done only for an unexecuted instruction whose destination register name matches a result register name held in R2. If the instruction is executed and its destination matches a register name in a result bundle, then the result value in the bundle is updated with the correct value of the destination register (from the instruction bundle). Also, on time 4, I2 meets R1 which has a register name that matches I2's destination name. The value for $\mathrm{r} 11$ in $\mathrm{R} 1$ is killed in this case. When I1 finishes execution on time 5, a new result bundle is injected that holds I1's destination value. The value is inserted to ensure that any subsequent dependent instruction can acquire its needed value. The destination value for I1 is copied to the instruction bundle for I1 so that the new value can be written to the register file when I1 reaches that stage 


\begin{tabular}{|l||c|c|c|c|c|c|c|c|c|}
\hline regfile & $\mathrm{R} 1$ & $\mathrm{R} 2$ & & & $\mathrm{R} 4$ & $\mathrm{I} 1$ & & & $\mathrm{R} 6$ \\
\hline logical & & $\mathrm{R} 1$ & $\mathrm{R} 2$ & $\mathrm{I} 1, \mathrm{R} 2 \mathrm{~K}$ & $\mathrm{I} 1, \mathrm{R} 3 \mathrm{I}$ & $\mathrm{R} 4$ & & & \\
\hline compare & & & $\mathrm{I} 1, \mathrm{R} 1 \mathrm{G}$ & & $\mathrm{R} 2$ & $\mathrm{R} 3$ & $\mathrm{R} 4$ & $\mathrm{R} 4$ & $\mathrm{I} 2, \mathrm{R} 4$ \\
\hline integer & & $\mathrm{I} 1$ & $\mathrm{I} 2$ & $\mathrm{I} 2, \mathrm{R} 1 \mathrm{~K}$ & $\mathrm{I} 2$ & $\mathrm{I} 2, \mathrm{R} 2 \mathrm{G}$ & $\mathrm{I} 2, \mathrm{R} 3 \mathrm{G}$ & $\mathrm{I} 2, \mathrm{R} 5 \mathrm{I}$ & $\mathrm{I} 3, \mathrm{R} 5$ \\
\hline regfetch & $\mathrm{I} 1$ & $\mathrm{I} 2$ & $\mathrm{I} 3$ & $\mathrm{I} 3$ & $\mathrm{I} 3, \mathrm{R} 1$ & $\mathrm{I} 3$ & $\mathrm{I} 3, \mathrm{R} 2$ & $\mathrm{I} 3, \mathrm{R} 3$ & $\mathrm{I} 4, \mathrm{R} 3$ \\
\hline fetch & $\mathrm{I} 2$ & $\mathrm{I} 3$ & $\mathrm{I} 4$ & $\mathrm{I} 4$ & $\mathrm{I} 4$ & $\mathrm{I} 4, \mathrm{R} 1$ & $\mathrm{I} 4$ & $\mathrm{I} 4, \mathrm{R} 2$ & $\mathrm{I} 5$ \\
\hline \hline
\end{tabular}

(a)

$$
\begin{aligned}
& \text { I1: } r[10]=r[11] \& r[12] \\
& \text { I2: } r[11]=r[10]+r[13] \\
& \text { I3: } r[20]=r[21]<=10 \\
& \text { I4: } r[21]=r[22]+r[23]
\end{aligned}
$$

(b)

$$
\begin{aligned}
& \mathrm{R} 1=<\mathrm{r} 11, \mathrm{r} 12>\text { (from regfile) } \\
& \mathrm{R} 2=<\mathrm{r} 10 . \mathrm{r} 13>\text { (from regfile) } \\
& \mathrm{R} 3=<\mathrm{r} 10>\text { (inserted by } \mathrm{I} 1) \\
& \mathrm{R} 4=<\mathrm{r} 21>\text { (from regfile) } \\
& \mathrm{R} 5=<\mathrm{r} 11>\text { (inserted by } \mathrm{I} 2)
\end{aligned}
$$

(c)

Fig. 2. Instruction and result movement in an example CFP. (a) Pipeline timing diagram (K = kill, $G=$ garner, I = insert). (b) Instruction sequence. (c) Result bundles.

(time 6). Result register names that match I1's destination register name can also be updated with the destination value from I1's instruction bundle. Notice that on time 5, I2 is stalled in the integer pipeline stage, where it executes. I2 will wait in this stage until it has acquired all of its source operand values. I2 garners its first operand, register 13, on time 6 from result R2. Because register 10 was killed in R2, I2 cannot copy the value for register 10 from the result. Instead, on time 7, I2 copies the value of register 10 from R3. On time 8, I2 executes and injects a new result bundle R5. Finally, on time 9, I2 advances to the next stage now that it has executed and a new instruction $\mathrm{I} 5$ is fetched.

A counterflow pipeline may also have pipelined functional units, called sidings, that execute instructions. Typically, the sidings execute high latency operations such as floating-point instructions and loads. Sidings are connected to the processor through launch and return stages, which initiate siding operations and return values from sidings. Fig. 1c shows an example siding for memory that is connected to the pipeline by memlaunch and memreturn stages. When a load instruction reaches memlaunch, it is launched to initiate a memory access. The load continues to advance through the main pipeline and, when it reaches memreturn, the loaded destination value is extracted from the memory siding. The destination value is copied to the load instruction bundle, and a new result bundle is injected into the result pipeline that holds the destination value.

\subsection{Design Characteristics of the Counterflow Pipeline}

As Fig. 1 shows, CFPs have local communication: Functional devices communicate only with their neighbors. This has two advantages. First, synthesis of CFPs does not need to determine device interconnection; it is implicit in pipeline stage order. Second, local communication may lead to fast and low power designs, especially as global wire delays dominate critical path latencies.
Although the simplicity and local communication of the CFP is appealing, there are some potential disadvantages [7]. First, the CFP requires arbitration between adjacent pipeline stages and, in practice, it has proven difficult to build fast (and correct) arbiters and control circuits for asynchronous implementations because of race conditions, circuit hazards, and handshaking. However, some designs have been proposed [13]. Second, enforcing the pipeline rules may be expensive because they require examining an instruction's state (e.g., whether it has been launched, executed, invalidated, etc.) and comparing an instruction's operands to a result bundle. Enforcing the pipeline rules may become a performance bottleneck because it affects instruction throughput and the speed at which results are sent to their consumer instructions. Finally, CFPs may use more chip area than traditional architectures because pipeline registers tend to be very wide.

\section{Wide Counterfol Pipelines}

Initially, we used the CFP for ASIP design, but found that the architecture could be improved considerably. In this section, we describe the shortcomings of the CFP for ASIPs and present our WCFP architecture, including several features required for high performance. Finally, we briefly describe our methodology to automatically generate WCFPs for kernel loops.

\subsection{CFP to WCFP}

The CFP issues one operation per instruction and tries to overlap the execution of multiple operations in separate pipeline stages to get good performance. The custom CFPs generated by our previous design techniques rely on hardware loop unrolling with speculative execution to get multiple loop iterations active in the pipeline at the same time. In practice, custom CFPs usually have only two iterations active at the same time, and in fact, frequently the $i+1$ th iteration only begins to execute while the $i$ th iteration 


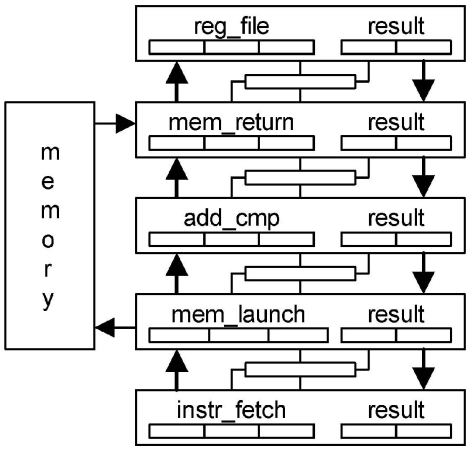

(a)

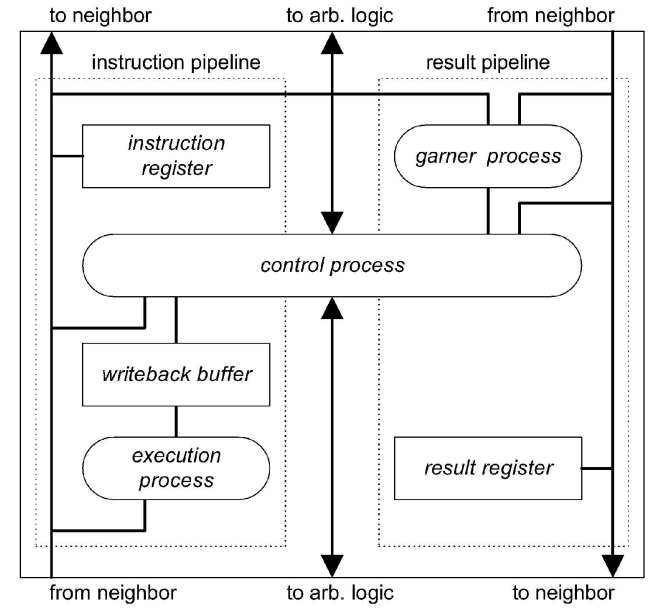

(b)

Fig. 3. A wide counterflow pipeline and a stage.

is executing. Data dependences between loop iterations may also limit the amount of execution overlap and the degree to which speculative execution can be applied across iterations. We found that this hardware unrolling was not sufficient to attain competitive levels of performance for ASIPs. To solve this problem and to make the CFP better suited for ASIP design, we extended the original proposal to a VLIW architecture that issues multiple operations per instruction. The WCFP increases instruction width so that multiple independent operations may be scheduled and executed simultaneously. The WCFP exploits ILP across multiple loop iterations by tailoring a custom WCFP to a software pipelined loop. By doing so, the WCFP better exploits ILP than the CFP.

Fig. 3a shows an example WCFP that issues three operations per instruction. The width of the result pipeline is matched to the maximum width of an instruction (in this example, the width is 2 since the add_cmp stage generates two results) to ensure there is enough space in a result bundle to convey all destination values of a wide instruction. There are no rules for what operations may be scheduled in an instruction; however, every operation in an instruction must use a different destination register, except for predicated operations that are guarded by complementary predicates. Although there is no explicit dependence checking, dependences between instructions are enforced inherently by the WCFP's pipeline rules. Restrictions such as issuing multiple memory operations together are determined by the operation repertoire of functional devices. For example, issuing two loads together requires a memory unit that can do two memory accesses simultaneously.

The operations in an instruction move through a WCFP in lock-step, although they may execute in different stages or sidings. For example, the operations in the instruction:

$$
\text { (ld [r5], r6; add } r 7,1, r 7 ; \operatorname{cmp~r10,0)}
$$

execute in two stages in Fig. 3a. The load is started in mem_launch and finished in mem_return and the addition and comparison are done in add_cmp.
Doing operations in separate stages lets them execute at the best point in the pipeline. The best location for an operation to execute is usually in the stage after the point where the operation acquires its last source operand. Because operations in an instruction may garner their operands in different stages and become ready to execute at different times, the location where each operation executes can be tailored to the data flow behavior of the application to improve performance.

\subsection{Pipeline Stage}

One issue with the design of the CFP is how to arrange pipeline stage control and processing. For the WCFP, we use a stage organization that is scalable with instruction width. Fig. $3 \mathrm{~b}$ shows a block diagram of control and processing in a WCFP execution stage. An execution stage has three processes: 1) garner, 2) execute, and 3) control. Garner checks for matching register names in an instruction and result bundle. If a match is found, the instruction and result are updated according to the CFP rules [1]. Execute checks whether an instruction executes in the stage. If it does, it waits until the operation it executes has all of its source operands before doing the operation. After executing the operation, a new result is generated and inserted into the result pipeline. Control monitors the activity of a stage and indicates to adjacent arbitration units whether it is ready to advance an instruction or result or accept a new instruction or result. The control process also moves instruction and result bundles between stages and inserts results generated by the execute process into the result pipeline. There is a writeback buffer in a pipeline stage to copy destination register values into the result pipeline. The buffer holds the value generated by the operations executed in a WCFP stage until it can be inserted into the result pipeline (see Section 3.4).

Included in the design of a WCFP stage is its local interconnection network. The interconnect must allow for comparing register names between an instruction and result bundle and for copying values to and from a result bundle. Fig. 4 shows one possible structure for comparing register 


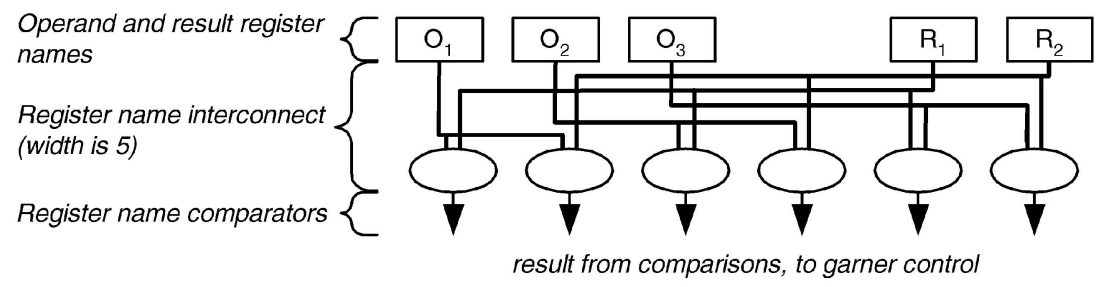

(a)

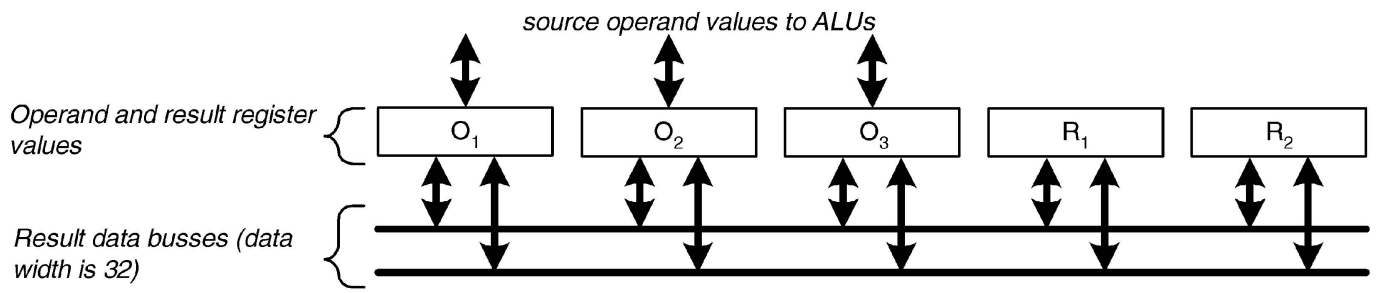

(b)

Fig. 4. Interconnect for a WCFP stage (On are operands and Rn are result registers). (a) Register name comparison network. (b) Result bus (shown for garnering).

names and copying and injecting result values. Fig. 4a shows the interconnect with comparators for checking an instruction's source register names against the names in a result bundle. To permit maximum parallelism, every instruction operand and result name have a comparator and there is a connection for each operand and result register name to the comparators. The figure shows three operands and two register names, with six comparators and five connections to the comparators. Similarly, Fig. $4 \mathrm{~b}$ shows the result buses needed for copying and updating register values. In this case, one bus is needed per value to copy or update a result value. In general, there are $n{ }^{*} m$ register name comparators, $n+m$ register name connections, and $m$ result buses in a stage, where $n$ is the number of operands in an instruction and $m$ is the number of results in a result bundle.

The design of a WCFP pipeline stage presents a possible limitation to the architecture. The control and interconnect in each stage that enforces the pipeline rules is potentially complex, especially for very wide pipelines. The size of the network is a concern because, as network size increases, the delay to determine a register name match and copy values also increases and a large network requires complex routing of instruction operands and results among the comparators. In a later section, we evaluate the impact of stage latency on overall performance. We also describe a way to quantitatively compare the size of the networks in evaluating the cost of WCFPs.

\subsection{Predication}

To reduce control transfer operations and to support aggressive ILP code transformations, the WCFP supports predicated execution. As demonstrated by other work [14] [15], this can expose significant ILP by flattening the control flow graph into a sequence of operations that can be scheduled together. The WCFP has guarded operations and predicate-generating comparisons similar to those of IMPACT EPIC [16] and HPL PlayDoh [17]. The WCFP handles predicated execution naturally by treating predicates as any other source operand and checking the value of the predicate before executing or launching an operation. Individual operations within an instruction are predicated; there is no instruction predicate that guards all operations in an instruction. For example, the instruction:

$$
\text { (1d_p p0, [r5], r6; add_p p1, r7, 1, r7; cmp r10,0) }
$$

has three operations, two of which are guarded by different predicates ( $1 d \_p$ and add_p). Predicating each operation gives maximum scheduling flexibility since operations with different predicates may be scheduled in the same instruction. Operations that are guarded by complementary predicates with the same destination register may also be scheduled in the same instruction.

The hardware support needed to add predicated execution to the WCFP is modest. First, there must be enough predicate and general-purpose registers to aggressively apply if-conversion. The WCFP has separate register files for predicate and general-purpose registers. The size of these files is adjusted by our synthesis system to meet the needs of a given kernel loop.

Second, execution units should not generate a result on a false predicate. Predicated operations are always executed, regardless of their predicate value. However, the value generated by an execution unit is inserted in the result pipeline only if the predicate is true. Otherwise, the generated value is ignored. Finally, the status of an operation's predicate must be checked before updating the register file with a destination register to ensure that the destination value is valid.

Predicated execution in the WCFP requires that the original value of the destination register be inserted into the result pipeline when a false predicate is encountered. This is done by including the destination register as a source operand to a predicated operation and by copying the original value of the destination register to the result pipeline on a false predicate. The garner process identifies this condition. The original value is inserted because dependent operations may be waiting for the destination 


\begin{tabular}{|l|}
\hline stage 3 \\
\hline stage 2 \\
\hline stage 1 \\
\hline
\end{tabular}

\begin{tabular}{|c|c|c|c|c|}
\hline $\mathrm{R} 1$ & & $\mathrm{I} 1$ & & \\
\hline $\mathrm{I} 1$ & $\mathrm{I} 1, \mathrm{R} 1$ & $\mathrm{R} 2$ & & \\
\hline $\mathrm{I} 2$ & $\mathrm{I} 2$ & $\mathrm{I} 2, \mathrm{R} 1$ & $\mathrm{I} 2, \mathrm{R} 2$ & $\mathrm{I} 2, \mathrm{R} 3$ \\
\hline \hline $\mathbf{0}$ & $\mathbf{1}$ & $\mathbf{2}$ & $\mathbf{3}$ & $\mathbf{4}$ \\
\hline
\end{tabular}

(a)

\begin{tabular}{|c|c|c|c|c|}
\hline $\mathrm{R} 1$ & & $\mathrm{I} 1$ & & \\
\hline $\mathrm{I} 1$ & $\mathrm{I} 1, \mathrm{R} 1$ & & & $\mathrm{I} 2$ \\
\hline $\mathrm{I} 2$ & $\mathrm{I} 2$ & $\mathrm{I} 2, \mathrm{R} 1$ & $\mathrm{I} 2, \mathrm{R} 2$ & \\
\hline \hline $\mathbf{0}$ & $\mathbf{1}$ & $\mathbf{2}$ & $\mathbf{3}$ & $\mathbf{4}$ \\
\hline
\end{tabular}

(b)

Fig. 5. Example of result injection (a) without and (b) with result packing.

register and, if a value were not generated, those operations would deadlock.

Although it is possible to squash operations with false predicates before they reach their execution point, the increase in stage complexity is usually not worth the small performance improvement. To squash predicated operations as soon as possible requires that all stages have the ability to recognize an operation with a false predicate and to insert the original destination value of the operation (contained in the instruction bundle) into the result pipeline. The pipelines in this paper support squashing operations with false predicates only at their execution point, which simplifies the WCFP hardware for stages that do not handle predicated operations.

An alternative WCFP predication scheme to the one proposed above inserts the destination register of a falsepredicate operation into the result pipeline when the operation flows through the register file. However, this scheme may substantially delay the delivery of source operands to dependent operations and, from experimental work, we have found that it is not competitive with the scheme described above. The pipelines in this paper use the first approach with a register cache at the beginning of the pipeline that holds register values from results that have recently exited the pipeline (see Section 3.5). In most cases, the previous value of the destination register is acquired from the register cache, which avoids requesting the destination register from the register file.

\subsection{Result Packing}

When an instruction executes in a WCFP stage, it needs to inject its destination values into the result pipeline. The manner in which this insertion is done can have an impact on pipeline performance. Our approach uses the writeback buffer in Fig. 3b to insert new register values into the result pipeline. The buffer holds destination values generated by executing an operation in the stage and the control process moves values from the buffer to the result pipeline. There are two feasible ways to do this. In the first choice, control waits until the result register is empty before moving the value from the writeback buffer to the result register. This inserts a new value into the stream of result bundles flowing through the result pipeline. This approach is relatively simple; however, it increases pressure on result pipeline bandwidth by generating new result packets for every destination register, which causes additional garner operations over the execution lifetime of a loop.

The second alternative moves the buffer's value immediately to the result register, regardless of whether the register is empty. When there is a bundle in the result register with enough space to hold the buffer's value, the value is packed into the existing bundle. If the bundle in the result register is full, the writeback buffer's value is retained until the full result bundle exits the stage. Once the full bundle exits, a new bundle is inserted containing only the buffer's value. Packing results limit the number of individual packets flowing through the result pipeline, which reduces the number of garner operations and improves result pipeline bandwidth utilization.

Fig. 5 shows how result packing helps performance. The example shows two instructions: I1 and I2, with I2 dependent on I1. The example assumes unit latency for the pipeline and a result pipeline width of two. The first instruction executes in stage 2 and injects the destination register value into the result pipeline at this stage. I2 executes in stage 1 and waits in that stage until I1 produces a result. The first diagram in Fig. 5a shows how result flow is affected by not packing results. In the figure, I1 finishes executing at time 1 and is ready to insert its destination in the result pipeline. However, the result registers in this stage are occupied by result bundle R1. Because results are not packed, the destination value generated by I1 cannot be injected until R1 has flowed through stage 2. At time 2, R1 leaves stage 2 and advances to next stage 1 . The destination value of I1 can be inserted in the result pipeline at this time and a new result bundle $\mathrm{R} 2$ is created to hold the destination value. R2 reaches I2 at time 3 and R2 executes and generates a result at time 4 .

Fig. 5b shows what happens when results are packed. On time 1, I1 generates a result and inserts it into result bundle $\mathrm{R} 1$. In this case, R1 has only one register value and can hold one more, so I1's destination value is packed into R1. At time 2, R1 flows to stage 1 and I2 acquires its dependent source operand from R1. Hence, on time 3, I2 executes and generates a new result. In this case, the result pipeline stage 1 is empty and a new result bundle, $R 2$, is injected to hold I2's destination value. The first example takes a total of five steps, while the second one takes four steps to do the same computation. Also, notice that one less result bundle has been inserted with result packing.

Although result packing can be applied to the CFP, it is particularly important for the WCFP. With the increased width of the WCFP's instruction pipeline, there needs to be a corresponding increase in the width of the result pipeline. That is, the width of the result pipeline must at least match the maximum number of destination values that can be generated in a stage. Furthermore, with more operations per instruction word, there is a greater need to deliver more 


\begin{tabular}{|c|c|c|c|c|c|c|}
\hline regfile & & & R6 & & I4 & \\
\hline stage 2 & & & & I4,R6 & & \\
\hline stage 1 & R5 & & I4 & I5 & I5,R6 & I5,R7 \\
\hline regfetch & & $\mathrm{I} 4, \mathrm{R} 5$ & I5,R5 & & & R6 \\
\hline \multirow[t]{2}{*}{ fetch } & $\mathrm{I} 4, \mathrm{R} 4$ & I5 & & $\mathrm{R} 5$ & & \\
\hline & 10 & 11 & 12 & 13 & 14 & 15 \\
\hline
\end{tabular}

(a)

\begin{tabular}{|c|c|c|c|c|}
\hline & & & & I4 \\
\hline & & & I4 & I5 \\
\hline R5 & & I4 & I5,R6 & \\
\hline & I4,R5 & I5,R5 & & R6 \\
\hline I4,R4 & I5 & & & \\
\hline \hline $\mathbf{1 0}$ & $\mathbf{1 1}$ & $\mathbf{1 2}$ & $\mathbf{1 3}$ & $\mathbf{1 4}$ \\
\hline
\end{tabular}

(b)

Fig. 6. Example of instruction and result movement (a) without and (b) with a register cache.

operand values to an instruction. Unlike the CFP, there is a larger variation in the number of results generated by instructions in a WCFP. Hence, the increased bandwidth in the result pipeline is more likely to go underutilized in the WCFP. Because there are "available slots" in result bundles already in the pipeline, it is desirable to pack newly generated values into these existing bundles. Packing can also reduce the number of comparisons done during a loop's execution. Every extra comparison may slow down the advancement of instructions and hurt performance. In complex pipelines, packing results can have a considerable performance advantage over not packing results, as shown later.

\subsection{Register Caching}

The CFP had its register file at the end of the pipeline; however, placing the register file at the end of the pipeline may lead to poor performance because every instruction entering the pipeline must request its source operands directly from the register file. The source operands for an instruction are forwarded to their consumer instructions via the result pipeline. This forwarding can hurt performance because instructions may stall waiting for their source operands and because there is an increase in the number of individual results flowing in the pipeline, which causes additional comparisons and resource conflicts for result registers. Although Sproull et al. suggest using a "register cache" at the beginning of the pipeline to mitigate this problem [1], they do not investigate the structure or performance of such a cache.

The WCFP keeps a cache of register values that have exited the pipeline in recent result bundles. In this way, the register cache serves as a future buffer from which instructions just entering the pipeline can acquire their source operands. The cache holds future values in the sense that it can cache values that have not yet been committed to the register file. The register cache is a stage that is located at the very beginning of the pipeline after instruction fetch. To maintain register cache consistency, whenever an instruction moves through the cache, the destination registers of the instruction are marked invalid in the cache. This ensures that subsequent instructions will not acquire an old value for a destination register of an instruction that is currently active in the pipeline.

Fig. 6 has timing diagrams that show how a register cache improves performance. In the figure, assume that I5 needs two source registers $\mathrm{r} 9$ and $\mathrm{r} 10$ and that result bundle $\mathrm{R} 4=[\mathrm{r} 9]$ and R5 $=$ [r10]. Consider Fig. 6a which does not have a register cache. On time 10, result $\mathrm{R} 4$ exits the pipeline and, on time 11, I5 is fetched. I5 flows to regfetch on time 12. It garners the value of $\mathrm{r} 10$ from R5 and requests source operand register $\mathrm{r} 9$ from the register file. In this example, result $\mathrm{R} 6=[\mathrm{r} 9]$ is inserted by the register file. I5 continues to advance through the pipeline and reaches stage 1, where it executes. However, because I5 has not garnered its operand $\mathrm{r} 9$, it stalls in stage 1 . Register $\mathrm{r} 9$ arrives at time 14 (in R6) and I5 garners r9's value and executes. It injects R7 to hold its destination value on the next step. Without a register cache, it takes I5 six steps to execute.

In the second timing diagram, Fig. $6 \mathrm{~b}$, regfetch has a register cache. In this example, when $\mathrm{R} 4$ reaches regfetch at time 9 (not shown), the value of $\mathrm{r} 9$ is copied to the cache. When I5 enters regfetch at time 12, it copies the value of r9. I5 also meets R5 in regfetch and garners r10. I5 advances to stage 1 on the next time step and begins execution immediately because it has both source operand values. In this second timing diagram, I5 takes four steps to execute.

There are two observations about this example. First, the value of register $\mathrm{r} 9$ was not "lost" in the second timing diagram when R4 exited the pipeline. Instead, the value was cached at the beginning of the pipeline. Second, there was one fewer register fetch in Fig. 6b, which reduces pipeline result pressure. Hence, register caches can improve performance by letting instructions acquire their source operands very early in the pipeline and by avoiding requests to the register file.

For the WCFP, we developed three alternative caches and investigated how these caches improve performance. The organizations we consider are a result cache (R), a passthrough cache (PT), and a result and pass-through cache $(\mathrm{R}+\mathrm{PT})$. The $\mathrm{R}$ cache tracks the most recent value of registers that have exited the result pipeline. There is a valid bit associated with each cache entry that indicates whether a particular register has recently been seen by the cache. The valid bits are set for every register in a result that flows through the cache and the bits are reset for each destination register in an instruction. An instruction with invalid source operands (in the cache) will request those operands from the register file (which acts as a "history buffer" of computation that has exited the pipeline). The PT cache, on the other hand, has a single bit for each register that indicates whether there is an instruction active in the pipeline that writes to that register (the pass-through cache is a scoreboard that tracks which registers will be set by active instructions). Pass-through bits are reset for every register contained in a result that enters the cache. The pass- 


Predicated operations
Functional devices tailored to benchmark
Register file size tailored to benchmark
$R+P T$ register cache, hit latency 5 and miss latency 3
Stages pack destination values into existing results
Data memory siding depth is 5 , latency is 25
Multiply siding depth is 5 , latency is 25
Operations in pipeline have latency of 5
Multiply \& memory can issue two operations
Result pipeline width is same as instruction width
Multi-beat execution model

Predicated operations

Register file size tailored to benchmark

$R+P T$ register cache, hit latency 5 and miss latency 3

Stages pack destination values into existing results

Data memory siding depth is 5 , latency is 25

Multiply siding depth is 5 , latency is 25

Operations in pipeline have latency of 5

Result pipeline width is same as instruction width

Multi-beat execution mode
Predicated operations

Functional devices tailored to benchmark

Register file size tailored to benchmark

Cycle time is equivalent to 5 time steps in the WCFP

Data memory access has five cycle latency

Multiply has five cycle latency

Arithmetic and logical operations 1 cycle latency

There are up to two memory units in a design

Functional units are fully pipelined

Fig. 7. Architecture characteristics: (a) WCFP architecture parameters, (b) VLIW architecture parameters.

through bit helps to avoid requesting source operands from the register file when there is an instruction in the pipeline that generates a particular operand. This reduces result pipeline pressure, which may improve performance. The final organization $(\mathrm{R}+\mathrm{PT})$ is a combination of the first two: It caches register values and tracks the status of destination registers. In the evaluation of the WCFP, we look at how each cache organization affects pipeline performance.

\subsection{Design Methodology}

WCFP architectural synthesis operates on predesigned functional devices such as pipeline stages and functional sidings. This design methodology is similar to methodologies for systems-on-a-chip [18] [19] which use well-defined and specified prebuilt blocks to construct single chip systems in a modular manner. The design space of WCFPs is defined by processor functionality and topology. Processor functionality is the type and number of devices in a pipeline and topology is the interconnection of those elements. Processor functionality is characterized by a design database of computational elements that indicates device type and semantics for each database entry.

The WCFP design system accepts an application program (in C) with its kernel loop annotated as an input to the code optimizer vpo [20], which compiles the application and transforms the loop using code optimizations such as strength reduction, induction variable elimination, global register allocation, loop invariant code motion, global common subexpression elimination, etc. vpo passes the optimized kernel loop to WCFP architectural synthesis, which selects and instantiates computational devices from the design database and derives the processor interconnection network.

The synthesis process has five steps [8]. The first synthesis step constructs a software pipeline loop using iterative modulo scheduling [21]. The software pipeline kernel specifies the operations and functional elements to include in a WCFP. Step 2 does pipeline extraction, which instantiates stages and sidings for kernel instructions from the design database. After pipeline extraction, Step 3 creates an instruction set architecture from the software pipeline kernel and the WCFP. Once the custom WCFP and ISA are determined, Step 4 generates the complete instruction schedule for the software pipeline kernel loop. The final synthesis step does pipeline refinement, which iteratively adjusts the order of pipeline stages to match the kernel loop's execution behavior. Stage order is refined using a simple heuristic that identifies locations in the pipeline with heavy resource contention and rearranges stages to reduce this contention. For our benchmarks in this paper, the time required by our synthesis system varies from 1 minute and 54 seconds to 22 minutes and 15 seconds, with an average of 8 minutes and 29 seconds (on a Sun $300 \mathrm{MHz}$ UltraSPARC 1). Although this design time does not include logic synthesis, it indicates that our methodology is feasible for quick turnaround design of embedded processors.

\section{WCFP Performance}

To investigate how the WCFP compares to other ASIPs, we compared it against other application-specific architectures using the CFP and conventional VLIWs.

\subsection{Evaluation Methodology}

To evaluate WCFPs, we use several common benchmarks, including loops extracted from the previously described embedded applications in MediaBench [10]. The benchmarks have integer versions of the Livermore loops $\mathrm{k} 1, \mathrm{k} 5$, $\mathrm{k} 7$, and $\mathrm{k} 12$, the finite impulse response filter ( $\mathrm{fir}$ ), vector dot product (dot), and four other kernels extracted from complete applications. These loops include the 2D discrete cosine transformation (dct) used in image compression and an implementation of the Floyd-Steinberg image dithering algorithm (dither). We also extracted the vector computation $a=b k \bmod d$ from RSA encryption (mexp). The final loop is GSM 6.10 speech decoding (gsm).

We built a microarchitecture simulator for studying WCFPs [9]. The simulator models WCFPs with varying computational latencies. To move an instruction or result between stages takes one time unit, to garner a result takes three time units, and to launch or return an instruction from a siding takes three time units [22]. To execute an operation such as an addition takes five time units. High latency operations are scaled relative to low latency ones, so an operation such as multiplication, assuming it is five times slower than addition, takes 25 time units. Fig. 7a shows the the parameters for our simulations. To evaluate the WCFP's 


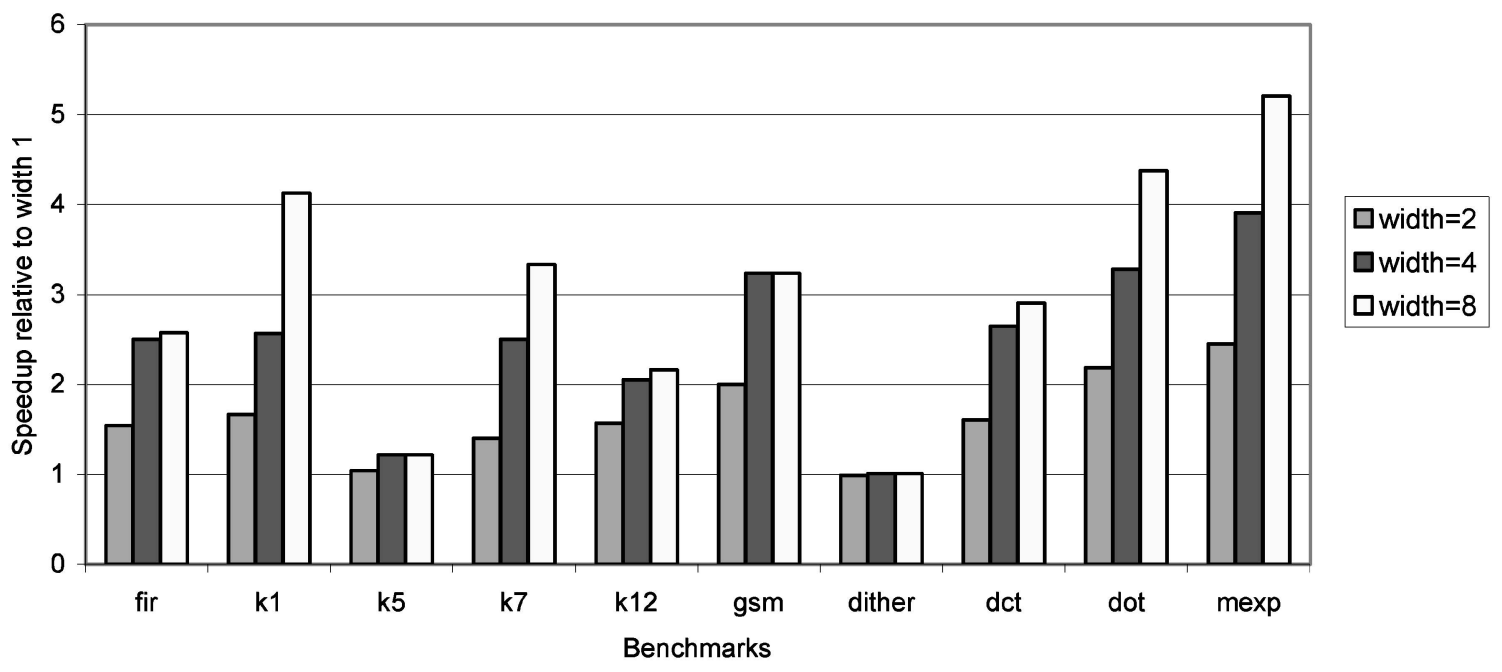

Fig. 8. Impact of instruction width.

performance, we use an instruction width of four in Sections 4.2, 4.3, and 4.4.

We also compare the WCFP to conventional VLIW architectures. For this comparison, we normalize execution latency of the WCFP relative to the VLIW. We assume that the clock cycle time of the VLIW is equivalent to five time steps in the WCFP. This assumption is based on the fact that simple operations, such as an ADD, take five time steps in the WCFP. We consider such simple operations to have the worst-case cycle time for the VLIW. Of course, in many architectures, operation latency does not dominate and other aspects determine clock cycle length, such as the L1 cache access time. Hence, normalizing to a simple operation conservatively evaluates the WCFP relative to the VLIW. Fig. 7b shows the architectural parameters for our VLIW design.

\subsection{Impact of Instruction Width}

To investigate how instruction width impacts performance, we synthesized different pipelines for the same benchmarks with different width constraints. Fig. 8 shows the speedup of custom WCFPs relative to custom CFPs for several benchmarks. The CFPs are similar to the WCFPs with the exception of pipeline width. They have the same register cache $(\mathrm{R}+\mathrm{PT})$ and functional repertoire as the WCFPs. In the figure, our synthesis tool was given maximum pipeline widths of one (i.e., a CFP), two, four, and eight operations. The widths correspond to design constraints that the synthesis system must maintain; it will not generate pipelines with operation widths greater than a given maximum width. However, based on the application code and its available instruction-level parallelism, pipelines with fewer operations can be generated.

The figure shows how performance is effected by a change in instruction pipeline width. For a width of two, there is a speedup of 1 to 2.45 times over a single-issue CFP. The average speedup in this case is 1.65 . For a width of four, the speedup is 1 to 3.9 times over the CFP (average is 2.5). Finally, for a width of eight, the speedups range from 1 to 5.2 times, with an average of 3.0. In the cases where an increase in pipeline width improves performance, there is also a corresponding increase in the average width of an instruction. For example, at a pipeline width of four, fir, $\mathrm{k} 1, \mathrm{k} 7$, gsm, dot, and mexp have speedups of 2.5-3.9 times greater than the CFP, which is approximately the same as the average increase in instruction width. For these benchmarks, the width of software pipeline kernel instructions is 2.7-3.8 operations. Other cases, such as $\mathrm{k} 5, \mathrm{k} 12$, and dither show smaller speedups with a change in pipeline width. In these cases, the speedup is constrained by instruction width, e.g., these benchmarks have 1.6, 2.2, and 2.52 operations per instruction for a pipeline width of four. Because these benchmarks have less ILP, wider pipelines have similar performance to narrower ones.

From the figure, we observe that an increase in performance follows an increase in instruction size and that the WCFP does not impose a performance penalty due to a change in instruction width. We also observe that benchmarks prefer different widths. For instance, fir, k5, and $\mathrm{k} 12$ achieve most of their speedup with a pipeline width of two. In these cases, increasing pipeline width has little benefit since the average kernel instruction size is not increased by relaxing the width constraint. This observation shows the importance of including design exploration to select a good pipeline width that will achieve a balance between performance and cost. In Section 5, we describe a cost model that can be used during design exploration to help guide selection of pipeline width.

\subsection{Impact of Stage Latency}

As described earlier, increasing stage width may adversely impact the latency of a stage. To get an indication of how increased stage latency harms performance, we varied the stage latency and measured its effect on overall performance, as shown in Fig. 9a.

The figure shows that, for a fixed instruction width, an increase in stage latency of up to 30 percent causes performance to be degraded by 7-13.5 percent. Fig. 9b shows average degradation for an increase in latency of 3-30 percent. An increase in latency of up to 30 percent causes a maximum average increase in execution latency of only 11.1 percent. This is very encouraging since the benchmark pipelines were not recustomized to take into 


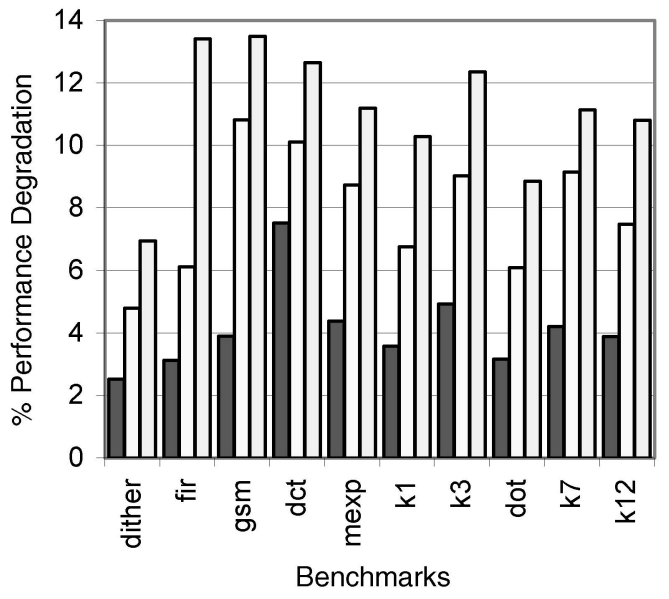

$10 \%$ Increase $\square 20 \%$ Increase $\square 30 \%$ Increase

(a)

Fig. 9. Effect of comparison latency on performance.

account the increase in comparison latency. Also, the instruction width was held constant and a wider instruction may improve overall performance by taking better advantage of ILP despite an increase in stage latency.

\subsection{Impact of Result Packing}

The WCFP packs destination register values into result bundles. To get an indication of how packing results improves performance, we measured the speedup of pipelines that pack results versus those that do not, as shown in Fig. 10a. The figure shows that, for most benchmarks, performance is improved significantly. For dct and k7, the speedup is 1.3. However, for two benchmarks, dither and $\mathrm{k} 5$, there was no performance improvement. Fig. 10b explains why performance is improved for each benchmark. The speedups come from the reduction in the number of garners done during a

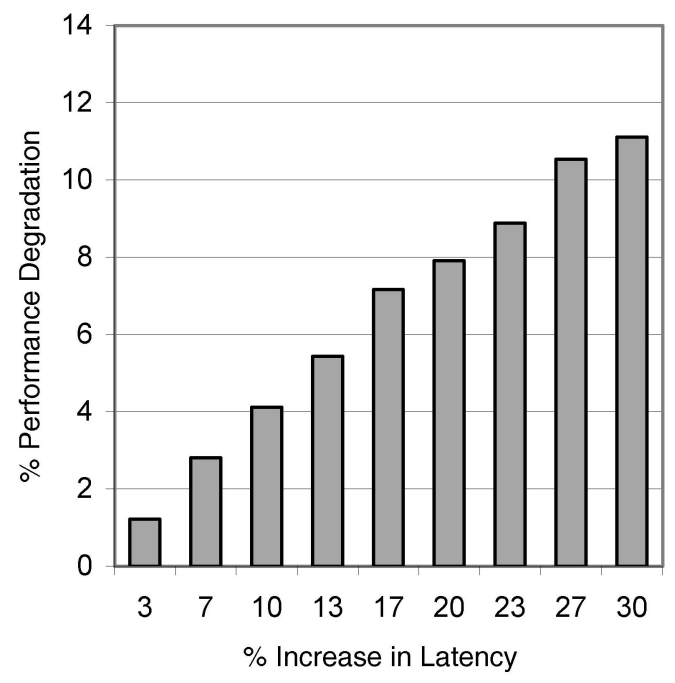

(b) benchmark's execution. For dither and k5, there is very little reduction in garners, i.e., the data flow for these benchmarks is such that few results are packed into existing result bundles. For dct and k7, many results are packed into existing bundles, which reduces the number of garners and improves performance. Packing results also reduce the number of garners for the other benchmarks; however, there is less of a relative speedup compared to dct and $\mathrm{k} 7$. The impact of reducing the number of garners is small because these benchmarks already do a good job of masking garner latency.

\subsection{Impact of Register Caching}

We also investigated how the $\mathrm{R}, \mathrm{PT}$, and $\mathrm{R}+\mathrm{PT}$ register cache organizations effect performance relative to baseline pipelines that do not have a register cache. Fig. 11a shows that a register cache can improve performance by up to 38 percent.

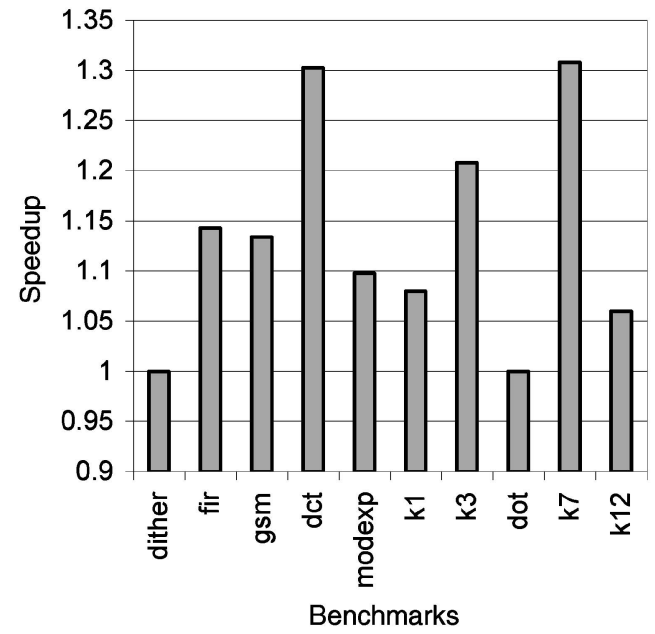

(a)

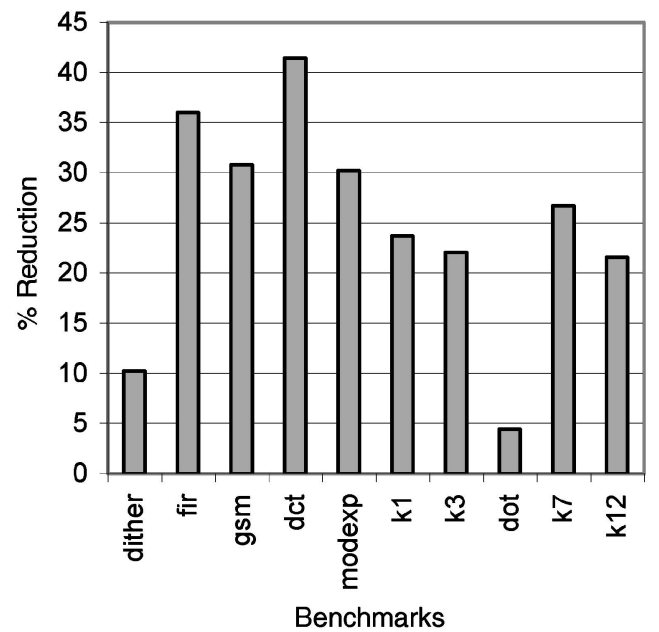

(b)

Fig. 10. Performance improvement of result packing. 


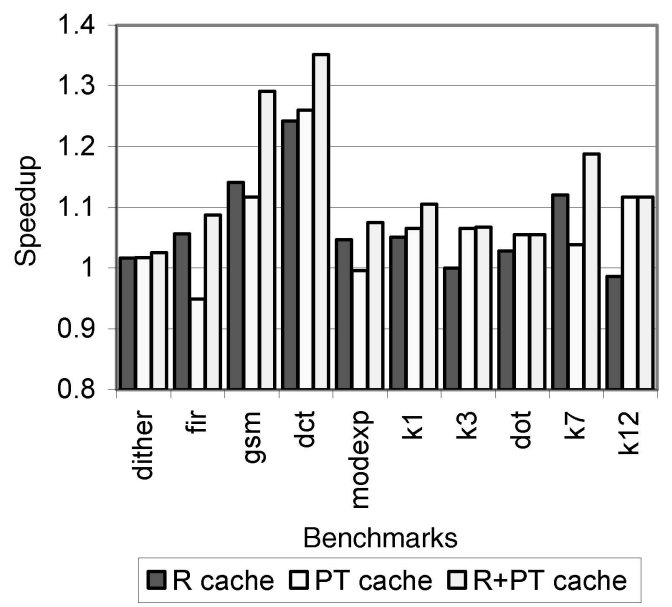

(a)

Fig. 11. Effect of different register caches on performance.

In a few cases, however, performance is not greatly affected; it is even degraded for $\mathrm{fir}$, modexp, and $\mathrm{k} 12$ (depending on the cache type). The performance degradation is due to the register cache causing pipeline stalls (while accessing the cache) early in the pipeline that affect the ability of instruction fetch to continue feeding the pipeline.

The figure also shows that the PT and R+PT organizations are the most effective at improving performance. Indeed, in many cases, the PT cache does surprisingly well. This good performance is especially encouraging since the PT cache has much less state per cache entry than the R+PT cache. The relative difference in performance between the $\mathrm{PT}$ and $\mathrm{R}+\mathrm{PT}$ organizations is an indication of the importance of reducing result pressure. That is, when there is little difference between PT and R+PT organizations (e.g., for dither, k1, k3, dot, and $\mathrm{k} 12$ ), the reduction in result pipeline pressure is more important than ensuring that an instruction acquires its source operands early in the pipeline. When the R+PT cache outperforms the PT cache (e.g., fir, gsm, dct, and k7), the early acquisition of source operands is more important than reducing result pipeline pressure.

Fig. $11 \mathrm{~b}$ shows the hit rates of each cache organization to get insight into why the caches help performance. For all of the cache organizations, a register cache hit means that a request for a source operand is not sent to the register file, which reduces the number of result bundles injected into the result pipeline by the register file. For the $\mathrm{R}$ cache, a hit means a source value is in the cache and it can be copied from the cache. For the PT cache, a hit is desirable because it means that a producer instruction is in-flight in the pipeline and a dependent instruction can pass through the register cache without requesting the in-flight source operand (it is guaranteed to be produced by the in-flight instruction). A hit in the R+PT cache combines the benefits of a hit in the R and PT caches.

The $\mathrm{R}$ cache has the lowest hit rate, as expected, ranging from $0.01-0.36$, with an average of 0.17 . The PT and R+PT caches have much higher hit rates that range from 0.8-0.98 (average is 0.88) and 0.98-0.99 (average is 0.99). The misses for the PT cache are associated with inactive destination

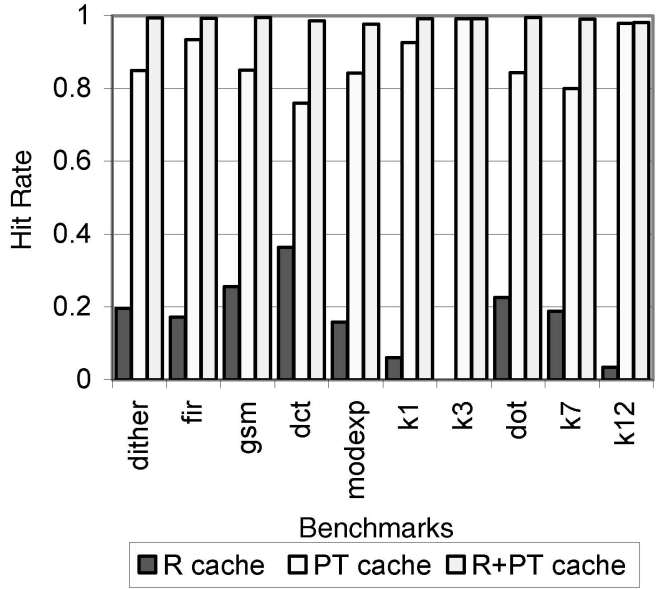

(b) registers and loop live-in and loop invariant registers, while the misses for the $\mathrm{R}+\mathrm{PT}$ cache are associated with cold start misses for loop live-in registers. The PT cache always misses for loop invariant registers since they are never updated by an instruction. The high hit rates associated with the PT and $\mathrm{R}+\mathrm{PT}$ caches indicate why performance is improved by these structures. The number of requests sent to the register file is much lower and there are fewer results flowing in the pipeline for the PT and R+PT caches than for the R cache.

\subsection{WCFP versus VLIW}

To evaluate the WCFP's performance relative to traditional VLIWs, we derived custom WCFPs and VLIWs tailored to the resource requirements of each benchmark. In this section, we summarize the results from Childers and Davidson [8] that compares WCFP and VLIW architectures. The custom VLIWs were tailored to the benchmark loops by varying the type and number of functional devices available so that operations could be scheduled in the software pipelined loop without concern for resource constraints other than instruction width. In all cases, the VLIWs and the WCFPs use the same instruction schedule to ensure a fair comparison between the two architectures.

The custom WCFPs achieve cycles per operation measurements that are competitive with traditional VLIWs with similar resource capabilities. Fig. 12 shows a comparison of effective cycles per operation for both VLIW and WCFP processors with varying instruction widths. We found that the WCFP is within 0-18 percent of the traditional VLIW's performance (the average is 8.6 percent) and, in most cases, performance is within 7 percent of the VLIW. For some cases, the WCFP does better than the VLIW. For example, the WCFP for $\mathrm{k} 5$ has 22 percent better performance than a custom VLIW. This benchmark has a good balance between the type of operations and the flow of instructions and results, which led to peak performance with minimal pipeline stalls.

The performance of the WCFP is encouraging because WCFP elements communicate only with their neighbors, unlike traditional VLIW architectures which 


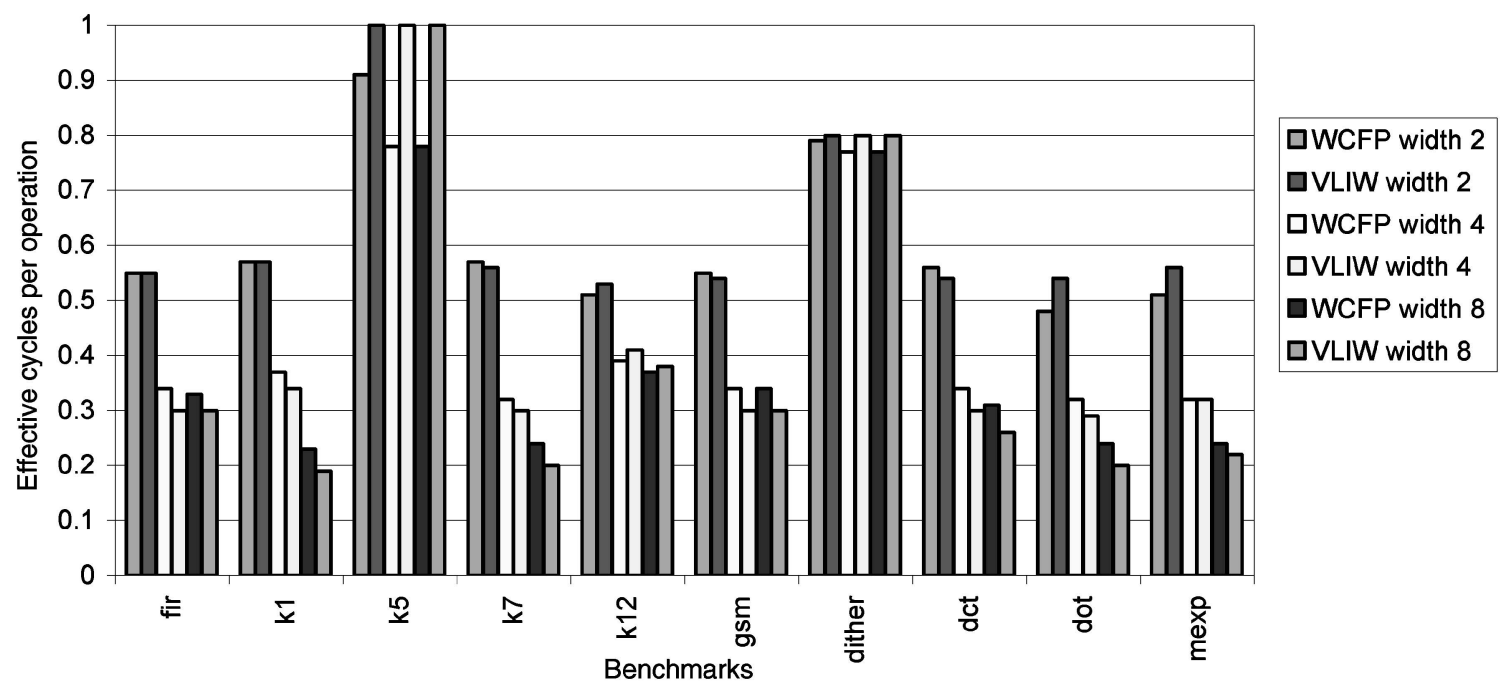

Fig. 12. Custom WCFPs versus custom VLIWs.

have complicated bus networks, pipeline bypasses, and other global structures to connect functional devices [4]. Also, as Flynn et al. [5] have observed, architectures with local communication will be especially important in the future as feature size continues to scale down into the deep submicron realm. Because a WCFP design system does not have to determine global interconnection of functional devices, a simple and effective design methodology may be used. Furthermore, the simplicity of the architecture and the design system does not come at the cost of performance of the generated pipeline designs.

\section{WCFP Cost}

In this section, we derive a storage-based cost model for CFP and WCFP architectures and evaluate the cost/ performance of the WCFP in comparison to the CFP. Importantly, the model can also be used to avoid selecting pipelines that violate a cost constraint or have poor cost/ performance.

\subsection{Cost Model}

To address the question of relative cost of WCFPs, we use a cost model based on the "bit width" of a pipeline stage. This model is derived from the microarchitecture design of pipeline stages implemented in our WCFP simulator. The model relies on approximations of the storage cost of state information within each pipeline stage, the register cache, and the register file. It also models the comparator network and local interconnect. The dominant cost in the model is instruction and result pipeline register cost since these registers tend to be very wide and account for most of the cost difference between alternative WCFP implementations derived with our methodology. To model the cost of instruction and result registers, we use bit width as the cost estimate since register size tracks bit width. The prototype implementation of our synthesis technique generates the same number and type of functional units for a particular input kernel loop code regardless of instruction width. Hence, the total cost of functional units is treated as a constant in the model. The cost of the comparator network is computed by multiplying the number of comparators by a constant cost for each comparator. A stage's local interconnect can also vary with a change in stage width. Our model handles the interconnect separately because it is difficult to determine the actual impact of the interconnect without a low-level implementation of a given pipeline. In the model, information about component costs are parameterized and can be refined to improve accuracy.

Instruction and Result Bundle Size. We start the discussion of the cost model with the bit width size of instruction and result bundles. These widths effect the amount of storage in the form of registers in each pipeline stage and are used in computing a pipeline's total cost. An approximation for the width of an instruction bundle register is:

$w_{\text {instr }}=\left(n_{\text {srcs }}+n_{\text {dests }}\right) \cdot\left(w_{\text {reg }}+w_{\text {spec }}+w_{\text {rflags }}\right)+w_{\text {op }}+w_{\text {flags }}$ where $n_{\text {srcs }}$ is the number of source operands (e.g., 2), $n_{\text {dests }}$ is the number of destination operands (e.g., 1), $w_{\text {reg }}$ is the width of a register value (e.g., 32), $w_{\text {spec }}$ is the width of a register name (e.g., 5), $w_{\text {rflags }}$ is the width of result flags (e.g., 2 for valid and garnered bits), $w_{o p}$ is the width of a decoded opcode (e.g., approximately 10 for a 12-stage pipeline), and $w_{\text {flags }}$ is the width of status flags (e.g., 2 for instruction killed and executed bits). As an example, a 32-bit triadic instruction requires a bundle width of $\left(n_{\text {srcs }}+\right.$ $\left.n_{\text {dests }}\right) \cdot\left(w_{\text {reg }}+w_{\text {spec }}\right)=3 \cdot(32+5)=111$ bits for just its source and destination operands. An approximation for the width of result registers is:

$$
w_{\text {result }}=n_{\text {result }} \cdot\left(w_{\text {reg }}+w_{\text {spec }}+w_{\text {rflags }}\right),
$$

where $n_{\text {result }}$ is the number of individual results in a result bundle.

Comparator Cost. In each pipeline stage, multiple comparators are needed to enforce the pipeline matching rules. The comparator network size varies with instruction and result bundle width. Although each of these comparators is small (their bit size is $w_{\text {spec }}$ ), a WCFP 


\begin{tabular}{|l||c|c|c|c|c|c|c|}
\hline \multirow{2}{*}{ Benchmark } & \multirow{2}{*}{ CFP Cost } & \multicolumn{6}{|c|}{ WCFP (Cost relative to custom CFP) } \\
\cline { 3 - 8 } & & \multicolumn{2}{|c|}{ Width 2 } & \multicolumn{2}{c|}{ Width 4 } & \multicolumn{2}{c|}{ Width 8 } \\
\hline & Pipeline & Pipeline & Interconnect & Pipeline & Interconnect & Pipeline & Interconnect \\
\hline \hline dither & 5,600 & 1.13 & $(1,2)$ & 1.62 & $(1,4)$ & 1.62 & $(1,4)$ \\
\hline fir & 5,517 & 1.29 & $(1,2)$ & 1.76 & $(1,4)$ & 2.33 & $(1.5,9)$ \\
\hline gsm & 6,569 & 1.22 & $(1,2)$ & 1.8 & $(1,4)$ & 2.09 & $(1,5)$ \\
\hline dct & 6,569 & 1.22 & $(1,2)$ & 2.42 & $(1,4.8)$ & 3.78 & $(2,16.8)$ \\
\hline mexp & 5,517 & 1.16 & $(1,2)$ & 2.11 & $(1.5,7.2)$ & 2.62 & $(1.5,10.8)$ \\
\hline$k 1$ & 5,254 & 1.28 & $(1,2)$ & 1.89 & $(2,8)$ & 2.75 & $(1.5,12.6)$ \\
\hline$k 5$ & 5,254 & 1.64 & $(1,2.4)$ & 2.18 & $(1,4.8)$ & 2.18 & $(1,4.8)$ \\
\hline dot & 4,088 & 1.25 & $(1,2$ & 1.87 & $(1.5,6)$ & 2.21 & $(1.5,9)$ \\
\hline$k 7$ & 7,095 & 1.42 & $(1,2.4)$ & 2.02 & $(1.5,7.2)$ & 3.14 & $(2,19.2)$ \\
\hline$k 12$ & 5,780 & 0.97 & $(1,2)$ & 1.84 & $(1.5,6)$ & 1.94 & $(1.5,7.5)$ \\
\hline \hline Average & 5,606 & 1.26 & $(1,2.1)$ & 1.95 & $(1.3,5.6)$ & 2.46 & $(1.5,9.9)$ \\
\hline
\end{tabular}

Fig. 13. Cost of sustom CFPs and WCFPs.

design may have: $n_{c m p}=\left(n_{\text {srcs }}+n_{\text {dests }}\right) \cdot n_{\text {result }}$ comparators per stage. We can estimate the cost of the comparator network as $c_{c m p}=w_{\text {spec }} \cdot n_{c m p} \cdot k_{c m p}$, where $k_{c m p}$ is a constant representing the per-bit area cost of a comparator. Typically, we set $k_{c m p}=1$ under the assumption that the per-bit cost of the comparator network is about the same as the per-bit cost of a pipeline register. For triadic instruction sets with $w_{\text {spec }}=5$ and $n_{\text {result }}=2$, this is approximately equivalent to one 32-bit comparator per stage $(3 \cdot 2 \cdot 5=30$ bits $)$.

Pipeline Storage Cost. Using $w_{i n s t r}, w_{\text {result }}$, and $c_{c m p}$, we can derive the cost of a pipeline stage. The cost is the sum of the bit width of the instruction and result pipelines, the comparator network, and the number of ALUs in a stage: The first term in this equation is the cost of the instruction and result registers. The cost of ALUs in a stage is given as the number of ALUs $\left(n_{A L U s}\right)$ multiplied by data width $\left(w_{\text {data }}\right)$. This estimate could be improved by factoring in the specific type of functional units in a stage and their actual cost from a design database. However, as mentioned above, the ALU cost is constant in our designs for a particular kernel loop regardless of instruction width. Hence, we treat ALU cost as a constant and set $w_{\text {data }}=32$. We estimate the cost of launch and return stages by setting $n_{A L U s}=0$. The cost of a WCFP includes the cost of functional sidings: $c_{\text {siding }}=n_{\text {ops }} \cdot n_{\text {stages }} \cdot w_{\text {data }}$. In this equation, $n_{\text {ops }}$ is the number of operations that can be issued at once (i.e., the number of pipelines in a siding) into a siding, $n_{\text {stages }}$ is the depth of the siding, and $w_{\text {data }}$ is the data width. The total cost of a pipeline is the sum of the cost of all stages and functional sidings: $c_{\text {pipeline }}=\sum c_{\text {stage }}+\sum c_{\text {siding }}$.

Register File and Register Cache Cost. The register file and register cache cost is the amount of total storage in each structure. For the register file, this cost, $c_{\text {regfile }}$, is simply the total number of registers multiplied by their bit width. For the register cache, the total cost, $c_{\text {regcache }}$, is dependent on the particular type of cache used. The R cache cost is the same as the register file cost with the addition of a valid bit. The PT cache has the cost of one bit per register name. Finally, the $\mathrm{R}+\mathrm{PT}$ cache is the sum of the $\mathrm{R}$ and PT cache costs. This storage model does not include the impact of multiple ports into the register cache and register file.

Total Cost. The pipeline cost equation, $c_{\text {pipeline, }}$ does not take into the account the cost of a pipeline's register file or register cache, although the size of these structures can be significant and varies with each custom WCFP. We can add in the cost of the register file and cache to get the total cost: $c_{\text {total }}=c_{\text {pipeline }}+c_{\text {regfile }}+c_{\text {regcache }}$.

Local Interconnect Cost. For the local interconnect, we consider it separately and do not include it in the total cost because the interconnect area does not directly follow the bit width of a stage. Instead, we determine the total number of local connections within a stage (based on Fig. 4). To simplify the estimate, we treat the local connections as buses. We compute two values for the local interconnect: the number of result data buses and the number of register name buses. The number of result buses is the maximum number of registers in a result bundle, $n_{\text {results }}$. We scale this number by the data width of each bus, which is $w_{\text {data }}$. Hence, the cost for the result buses in a stage is $c_{\text {resbus }}=n_{\text {results }} \cdot w_{\text {data }}$. The cost of the register name comparator interconnect can be computed in a similar manner. Here, the number of buses is $\left(n_{\text {srcs }}+n_{\text {dests }}\right) \cdot n_{\text {result }}$, which is $n_{\text {cmp }}$. To get the cost of the register name interconnect, we use $c_{\text {namebus }}=n_{c m p} \cdot w_{\text {spec }}$ because each bus is $w_{\text {spec }}$ bits wide.

\subsection{WCFP and CFP Cost Comparison}

Fig. 13 shows the cost of custom CFP and WCFPs with varying pipeline widths $(2,4$, and 8$)$ for several benchmarks. The width of each pipeline is a constraint on the maximum instruction width that can be generated by our synthesis system. The cost was computed using $c_{\text {total }}$ with $w_{\text {data }}=32, w_{r f l a g s}=2$, and $w_{\text {flags }}=2$. The size of the register file generated for each pipeline determines $w_{\text {spec }}$ for a pipeline. The table shows the cost of four pipelines for each benchmark and the relative increase in cost of custom WCFPs versus custom CFPs in the shaded regions of the table. For example, a WCFP that has an instruction width of 2 increases total cost by 1.21 times over a CFP. The table also shows the relative increase in cost of a stage's local interconnect. 


\begin{tabular}{|l||c|}
\hline Benchmark & Cost/Performance \\
\hline dither & 0.63 \\
\hline fir & 1.42 \\
\hline gsm & 1.8 \\
\hline dct & 1.1 \\
\hline mexp & 1.85 \\
\hline
\end{tabular}

\begin{tabular}{|l||c|}
\hline Benchmark & Cost/Performance \\
\hline$k I$ & 1.38 \\
\hline$k 5$ & 0.56 \\
\hline dot & 1.75 \\
\hline$k 7$ & 1.24 \\
\hline$k 12$ & 1.12 \\
\hline
\end{tabular}

Fig. 14. Cost/performance for instruction width of four.

Fig. 13 shows that the custom CFPs have costs ranging from 4,088 to 7,095 and WCFPs have costs ranging from 4,458 to 24,846 . The table gives insight into the relative cost increase between CFPs and WCFPs. From the table, a WCFP with a width of two increases cost by 0.98 to 1.64 times, with an average of 1.26 times. In the case of $\mathrm{k} 12$, the cost of the dual-issue WCFP is lower than the cost of the CFP. This is due to a decrease in pipeline length and the number of instruction and result registers. Although the WCFP and the CFP have the same number of ALUs, the WCFP has a shorter pipeline because it issues more operations per instruction. In the other cases, pipeline length does not decrease enough to reduce the overall cost. For many benchmarks, the CFP and the dual-issue WCFP have approximately the same length. This is due to the restriction that launch and return stages cannot be combined with other stages. That is, if there is an attached memory unit to a WCFP, then the memory launch and return stages must be separate and cannot be combined with other execution or launch and return stages. Therefore, because pipeline width increases and pipeline length stays the same relative to the CFP, the cost of the WCFPs increase.

Fig. 13 shows that the cost of the WCFPs does not increase at the same rate as width relative to the CFP. For example, dual-issue WCFPs do not have twice the cost of single-issue CFPs. This is due to several reasons. First, the number of ALUs and functional sidings stays the same across all of the architectures. Second, the result pipeline does not increase at the same rate as the instruction pipeline. The custom CFPs have a result pipeline width of two, which is the same as the dual-issue WCFPs. Result pipeline width does increase for WCFPs with instruction widths of four and eight, although not at the same rate as the instruction width. For an instruction width of two, most of the pipelines have a result width of three, i.e., the maximum number of operations done by any execution stage is three, and result pipeline width is set to this size. Finally, the increase in cost comes primarily from the increase in the width of registers in each stage and the increase in register file and cache size. Pipeline length does not decrease enough to offset these larger structures.

Fig. 13 also shows how an increase in width impacts a pipeline stage's local interconnect. For each benchmark and width, the table shows for the increase in cost of the result and register name buses as a tuple. The first element of the tuple is the increase in $c_{\text {resbus }}$ and the second element is the increase in $c_{\text {namebus }}$ over the baseline CFP. For a width of two, the table shows that the result bus interconnect does not increase in cost. In this case, all generated WCFPs have a result width of two, which is the same as the CFP. However, at pipeline widths four and eight, there is an increase in $c_{\text {resbus }}$ because the result pipeline width increased. The relative cost increase ranges from 1.0 to 2.0, with an average of 1.3. As noted earlier, the width of the result pipeline does not necessarily scale with an increase in instruction width and the result bus cost reflects the actual width. For example, at a width of four, $\mathrm{k} 7$ has a result width of three and a relative cost of 1.5 .

The table shows that the average relative cost of the register name buses is $2.1,5$, and 9.9 for widths of two, four, and eight. In some cases, the register name bus cost does not increase. For example, dither has the same cost at widths four and eight. Here, the generated pipelines are the same in both cases. Other benchmarks show a significant increase in cost from one width to another. For a width of eight, dct has a 16.8 times increase in $c_{\text {namebus }}$. There are two reasons for an increase in $c_{\text {namebus }}$. First, as width increases, there are more comparators and register name buses per stage (see Section 3.2). Second, for some benchmarks, the size of the register file increases with a change in width. For $k 1$, the size of the register file doubles when going from a width of four to eight. Because there are more registers, the size of $w_{\text {spec }}$ increases, which impacts $c_{\text {namebus }}$.

Fig. 14 shows the ratio of speedup to cost for custom WCFPs with an instruction width of 4 versus custom CFPs. This ratio illustrates whether "spending" additional chip area on a wide counterflow pipeline is worth the increase in performance. In general, the additional performance obtained from a custom WCFP versus a CFP should come at an equivalent (or smaller) cost in chip area. This implies that the speedup/cost ratio should be greater than 1 and most of the benchmarks in Fig. 14 have a speedup/cost ratio that is greater than 1 (the average is 1.28). fir, gsm, $\mathrm{k} 1$, dot, and mexp have ratios that are above 1 . In these cases, the additional cost of a WCFP is worth the increase in performance. Two of the benchmarks, dither and $\mathrm{k} 5$, have speedup/cost ratios of 0.63 and 0.56 . As indicated in Fig. 8, custom WCFPs for these benchmarks do not significantly improve performance due to the benchmarks' limited instruction-level parallelism. For dither and k5, the cost of WCFPs is not worth the small increase in performance. On average, we found that a quad-issue WCFP increases cost by an average of 1.95 times over the CFP, while performance increases an average of 2.5. From these results, we conclude that the WCFP is cost effective since performance is usually improved significantly for a small increase in cost over the CFP.

In a manner similar to the analysis above, our model can be used to select among WCFPs for a given benchmark. The model can be combined with performance estimates to guide a search for the best design alternative given cost and performance constraints. For example, dct has a 12 percent 
improvement in performance when going from an instruction width of four to eight. However, the pipeline cost for det increases by 57 percent and the interconnect cost increases by 100 percent for $c_{\text {resbus }}$ and 250 percent for $c_{\text {namebus. }}$. For dct, the 12 percent performance increase is probably not worth the increased cost.

\section{Related Work}

There has been much interest in automated design of ASIPs because of the increasing importance of high-performance and quick turnaround design in embedded systems. ASIP techniques typically address two broad problems: ISA and microarchitecture synthesis. ISA synthesis combines microoperations in a programs to create instructions [23] while meeting code size and execution latency constraints. Microarchitecture synthesis derives a processor from an application code. This work has particularly focused on developing computing architectures that can be dynamically tailored to the needs of an application, including Garp [24], Raw [25], PRISC [26], [27], DISC [28], RaPiD [29], [11], [30], CHESS [31], SPLASH [32], [33], [34], PAM [35], PipeRench [36], [12], and PICO [37], [38], [39], [40], [41].

SPLASH was one of the earliest reconfigurable computing systems [34]. More recent efforts, such as RaPiD and PipeRench, use a "chunky" architecture: Large macrocells can be configured and interconnected to form a custom system [42]. RaPiD uses a linear array of processing elements with pipelined control [29], [11], [30] and PipeRench uses an array of globally connected processing stripes that can be interconnected to form a virtual pipeline of any length [36], [12].

Another approach is based on architecture templates [43], [44], [45], [23]. The template parameterizes an architecture: The number and type of functional units and the interconnection topology can be customized by changing architectural parameters. Template architectures have often used a VLIW organization [44], [46], [47], [48]. One approach tailors a VLIW to the resource requirements of embedded loops [46]. The target architecture is a parameterized VLIW that allows changing the number of ALUs, operation repertoire, memory configuration, and register file size and cluster geometry. To synthesize a processor, they search the design space of all configurations, given constraints, and pick the one that best meets cost-performance goals.

PICO generates a computing system from an application's source code using both a custom VLIW and systolic array [37], [38], [39], [40], [41]. The systolic array is generated for an application's computationally expensive loop nest and a VLIW is generated for the control code. The systolic array is derived by applying systolic optimizations and software pipelining from which a processor is determined. Similarly, the VLIW is synthesized given certain constraints. The ShiftQ schema included in PICO is a buffered interconnect for functional units [40] that has similar advantages to the WCFP's results pipeline. The WCFP is attractive because it is composable and simple and yet it achieves performance competitive with other VLIW customization techniques, such as PICO.
Another template system is MOVE [49], [4]. This system uses transport triggered architectures (TTAs) that address the problems associated with functional unit and register file interconnection in VLIWs, particularly problems with scalability, performance, and cost of multiported register files. Similar to other systems, MOVE uses compiler technology and design exploration to derive a processor that meets an application's cost and computational requirements.

The CFP has been the focus of much research. Most past work has focused on implementation and proofs of correctness [50], [51], [52], [53], [54]. Although the CFP was proposed as an asynchronous organization [1], there has been work on a synchronous version [55], [56]. However, this work adds significant structures to the original design to get good performance on a variety of programs. In our work, we customize WCFPs to an application to get high performance without introducing expensive new features such as explicit register renaming.

\section{SUMmarY}

This paper describes a novel application of the CFP to automatic ASIP design. In the paper, we modify and extend the CFP to a wide-issue CFP that is easily customized to the resource and data flow requirements of embedded kernel loops. The paper describes the capabilities that we add to the CFP to better exploit instruction-level parallelism, including wide instructions, result packing, register caching, and predicated execution. The first capability executes multiple operations per instruction. Increasing instruction width has little impact on the CFP architecture, i.e., the CFP's basic operation and policies do not need to be changed. The primary concern with a wide pipeline stage is a wide stage has a larger interconnection network than a narrow one. Although an increase in stage width may adversely effect performance, we found that an increase in stage latency of up to 30 percent decreases performance by an average of only 11 percent. This performance degradation may be offset by executing more operations per instruction. The second capability that we add is result packing, which inserts newly generated destination values into already existing result bundles. Result packing reduces the number of results flowing in a pipeline and the number of distinct garner operations done during the execution of a loop. It reduces the number of distinct garner operations by 4-41 percent, with an average of 26 percent, and improves performance by up to 30 percent, with an average of 14 percent. A third capability puts a register cache at the beginning of a WCFP to hold results that have recently exited the result pipeline. We propose three types of register caches (a result cache, pass-through cache, and a passthrough cache with result cache) that effectively improve performance by an average of 14 percent due to high register cache hit rates of up to 99 percent. The paper describes a final enhancement for predication in WCFPs. Predicated execution requires minimal hardware: the instruction set needs guarded operations and predicategenerating comparisons; functional units must be disabled for operations with false predicates; and operations must insert their original destination register's value into the result pipeline on a false predicate. 
The WCFP lends itself well to automatic ASIP design. Custom WCFPs outperform CFPs by an average of 2.5 times. We also show in the paper, using an analytic cost model, that the increased performance of the WCFP does not come at a significantly increased cost in comparison to the CFP. Most importantly, custom WCFPs are competitive with VLIWs that have been tailored to the resource requirements of embedded loops, without requiring expensive and complicated global interconnection of functional units, register files, dispatch logic, etc. This has two important advantages. First, as global wire delays dominate circuit latency, the WCFP's local communication should lead to very fast implementations. Second, the WCFP's local communication reduces the complexity of automatic ASIP design because synthesis does not have to derive bus networks or other global structures. Based on these observations and the results presented in the paper, the WCFP is well-suited for automatic and quick turnaround design of custom embedded processors.

\section{ACKNOWLEDGMENTS}

The authors would like to thank the reviewers for their careful reading and helpful comments on the initial manuscript.

\section{REFERENCES}

[1] R.F. Sproull, I.E. Sutherland, and C.E. Molnar, "The Counterflow Pipeline Processor Architecture," IEEE Design and Test of Computers, vol. 11, no. 3, pp. 48-59, 1994.

[2] S.P.E. Corp., "Cpu2000 Benchmark Suite,"www.spec.org, 2000.

[3] M. Schlett, "Trends in Embedded-Microprocessor Design," Computer, pp. 44-49, Aug. 1998.

[4] H. Corporaal, Microprocessor Architecture from VLIW to TTA. John Wiley \& Sons, 1998.

[5] M.J. Flynn, P. Hung, and K.W. Rudd, "Deep Submicron Microprocessor Design Issues," IEEE Micro, vol. 19, no. 4, pp. 11-22, July 1999.

[6] B.R. Childers and J.W. Davidson, "A Design Environment for Counterflow Pipeline Synthesis," Proc. ACM SIGPLAN Workshop Languages, Compilers, and Tools for Embedded Systems, June 1998.

[7] B.R. Childers and J.W. Davidson, "Architectural Considerations for Application-Specific Counterflow Pipelines," Proc. 20th Conf. Advanced Research in VLSI, Mar. 1999.

[8] B.R. Childers and J.W. Davidson, "Automatic Architectural Design of Wide-Issue Counterflow Pipelines," Proc. Workshop Compilers and Architectures for Embedded Systems, Oct. 1999.

[9] B.R. Childers and J.W. Davidson, "An Infrastructure for Designing Custom Embedded Counterflow Pipelines," Proc. Hawaii Int'l Conf. System Sciences, Jan. 2000.

[10] C. Lee, M. Potkonjak, and W.H. Magione-Smith, "Mediabench: A Tool for Evaluating and Synthesizing Multimedia and Communications Systems," Proc. 30th Symp. Microarchitecture, pp. 330-335, Dec. 1997.

[11] C. Ebeling, D.C. Cronquiest, and P. Franklin, "Mapping Applications to the Rapid Configurable Architecture," Proc. Fifth Ann. Symp. Field-Programmable Custom Computing Machines, pp. 106-115, Apr. 1997.

[12] S.C. Goldstein, H. Schmit, and M. Moe, "Piperench: A Coprocessor for Streaming Multimedia Acceleration," Proc. 26th ACM Int'l Symp. Computer Architecture, pp. 28-39, May 1999.

[13] W.H. Korver and I.M. Nedelchev, "Asynchronous Implementation of the SCPP: A Counterflow Pipeline Processor," IEE Proc. Computers and Digital Techniques, vol. 143, no. 5, pp. 287-294, 1996.

[14] S.A. Mahlke, R.E. Hank, and R.A. Bringmann, "Characterizing the Impact of Predicated Execution on Branch Prediction," Proc. 27th Symp. Microarchitecture, pp. 217-227, Dec. 1994.

[15] S.A. Mahlke, R.E. Hank, and J.E. McCormick, "A Comparison of Full and Partial Predicated Execution Support for ILP Processors," Proc. 22nd Int'l Symp. Computer Architecture, pp. 138-149, June 1995.
[16] D.I. August, K.M. Crozier, and P.R. Sias, "The Impact Epic 1.0 Architecture and Instruction Set Reference Manual," Technical Report IMPACT-98-04, Univ. of Illinois, Feb. 1998.

[17] V. Kathail, M.S. Schlansker, and B.R. Rau, "HPL Playdoh Architecture Specification: Version 1," Technical Report HPL-9380, HP Labs, Feb. 1994.

[18] H. Chang, L. Cooke, M. Hunt, G. Martin, A. McNelly, and L. Todd, Surviving the SOC Revoluation: A Guide to Platform-Based Design. Kluwer Academic, 1999.

[19] M. Keating and P. Bricaud, Reuse Methodology Manual: For Systemson-a-Chip Designs. Academic Publishers, 1999.

[20] M.E. Benitez and J.W. Davidson, "A Portable Global Optimizer and Linker," Proc. ACM Programming Language Design and Implementation, pp. 329-338, June 1988.

[21] B.R. Rau, "Iterative Modulo Scheduling: An Algorithm for Software PipelinedLoops," Proc. 27th Int'l Symp. Microarchitecture, pp. 63-74, Dec. 1994.

[22] I.E. Sutherland, E-mail communication and discussion on CFP implementation, 1994.

[23] I.J. Huang and A.M. Despain, "Synthesis of Application Specific Instruction Sets," IEEE Trans. Computer-Aided Design of Integrated Circuits and Systems, vol. 14, no. 6, pp. 663-675, June 1995.

[24] J.R. Hauser and J. Wawrzynek, "Garp: A MIPS Processor with Reconfigurable Coprocessor," Proc. IEEE Fifth Symp. Field-Programmable Custom Computing Machines, pp. 12-21, Apr. 1997.

[25] E. Waingold, S. Devabhaktuni, and V. Sarkar, "Baring It All to Software: Raw Machines," Computer, vol. 30, no. 9, pp. 86-93, Sept. 1997.

[26] R. Razdan and M.D. Smith, "A High-Performance Microarchitecture with Hardware-Programmable Functional Units," Proc. 27th Int'l Symp. Microarchitecture, pp. 172-180, Dec. 1994.

[27] R. Razdan, "PRISC: Programmable Reduced Instruction Set Computers," PhD dissertation, Harvard Univ., May 1994.

[28] M.J. Wirthlin and B.L. Hutchings, "A Dynamic Instruction Set Computer," Proc. IEEE Workshop FPGAs for Custom Computing Machines, pp. 99-107, Apr. 1995.

[29] D. Cronquist, P. Franklin, S. Berg, and C. Ebling, "Specifying and Compiling Applications for Rapid," Proc. IEEE Workshop FPGAs for Custom Computing Machines, pp. 116-127, Apr. 1998.

[30] C. Ebeling, D.C. Cronquist, P. Franklin, and C. Fisher, "Rapid: A Configurable Computing Architecture for Compute-Intensive Applications," Proc. Field Programmable Logic and Applications, pp. 126-135, 1997.

[31] A. Marshall, T. Stansfeld, and I. Kostarnov, "A Reconfigurable Arithmetic Array for Multimedia Applications," Proc. Seventh Int'l Symp. Field-Programmable Gate Arrays, pp. 135-143, Feb. 1999.

[32] J.M. Arnold, D.A. Buell, and E.G. Davis, "Splash 2," Proc. Fourth Ann. ACM Symp. Parallel Algorithms and Architectures, pp. 316-324, June 1992.

[33] J.M. Arnold, "The Splash 2 Software Environment," Proc. IEEE Workshop FPGAs for Custom Computing Machines, pp. 88-93, Apr. 1993.

[34] D.A. Buell, J.M. Arnold, and W.J. Kleinfelder, Splash 2: FPGAs in Custom Computing Machines. IEEE CS Press, 1996.

[35] P. Bertin and H. Tovati, "Pam Programming Environments: Practice and Experience," Proc. IEEE Workshop FPGAs for Custom Computing Machines, pp. 133-138, Apr. 1994.

[36] S. Cadambi, J. Weener, and S.C. Goldstein, "Managing Pipeline Reconfigurable FPGAs," Proc. Sixth Int'l Symp. Field-Programmable Gate Arrays, pp. 55-64, Feb. 1998.

[37] V. Kathail, S. Aditya, R. Schreiber, B. Rau, D. Cronquist, and M. Sivaraman, "Pico: Automatically Designing Custom Computers," Computer, vol. 35, no. 9, pp. 39-47, Sept. 2002.

[38] S. Aditya, R. Rau, and V. Kathail, "Automatic Architectural Synthesis of VLIW and Epic Processors," Proc. IEEE Int'l Symp. System Synthesis, pp. 107-113, Nov. 1999.

[39] R. Schreiber, S. Aditya, and R. Rau, "High-Level Synthesis of Nonprogrammable Hardware Accelerators," Proc. IEEE Conf. Application-Specific Systems, Architectures, and Processors, pp. 113124, July 2000.

[40] S. Aditya and M. Schlansker, "Shiftq: A Buffered Interconnect for Custom Loop Accelerators," Proc. Conf. Compilers, Architectures, and Synthesis for Embedded Systems, pp. 158-167, Nov. 2001.

[41] S. Mahlke, R. Ravindran, M. Schlansker, R. Schreiber, and T. Sherwood, "Bitwidth Cognizant Architecture Synthesis of Custom Hardware Accelerators," IEEE Trans. Computer-Aided Design, Nov. 2001. 
[42] J. Villasenor and W.H. Mangione-Smith, "Configurable Computing," Scientific Am., vol. 276, no. 6, pp. 54-59, June 1997.

[43] M. Auguin, F. Boeri, and C. Carriere, "Automatic Exploration of VLIW Processor Architectures from a Designers Experience Based Specification," Proc. Workshop Hardware Software Codesign, pp. 108115, Sept. 1994.

[44] A. DeGloria and P. Faraboschi, "An Evaluation System for Application Specific Architectures," Proc. 23rd Int'l Workshop Microarchitecture and Microprogramming, pp. 80-89, Nov. 1990.

[45] B. Holmer and A. Despain, "Viewing Instruction Set Design as an Optimization Problem," Proc. 24th Int'l Workshop Microprogramming and Microarchitecture, pp. 153-162, Nov. 1991.

[46] J.A. Fisher, P. Faraboschi, and G. Desoli, "Custom-Fit Processors: Letting Applications Define Architectures," Technical Report HP45-96-144, HP Labs, 1996.

[47] J.M. Mulder, R.J. Portier, A. Srivastava, and R. Velt, "An Architecture Framework for Application-Specific and Scalable Architectures," Proc. 16th Int'l Symp. Computer Architecture, pp. 362-369, May 1989.

[48] A. Wu and W. Wolf, "Data-Path Synthesis of VLIW Video Signal Processors," Proc. 11th Int'l Symp. System Synthesis, pp. 96-101, Dec. 1998.

[49] H. Corporaal and J. Hoogerbrugge, "Cosynthesis with the Move Framework," Proc. Symp. Modelling, Analysis, and Simulation, pp. 184-189, July 1996

[50] B. Coates, J. Ebergen, and J. Lexau, "A Counterflow Pipeline Experiment," Proc. Int'l Conf Advanced Research in Asychronous Circuits and Systems, pp. 161-172, Apr. 1999.

[51] P.N. Loewenstein, "Formal Verification of Counterflow Pipeline Architecture," Proc. Eighth Int'l Workshop Higher Order Logic Theorem Proving and Its Applications, pp. 261-276, Sept. 1995.

[52] M.B. Josephs, P.G. Lucassen, J.T. Udding, and T. Verhoeff "Formal Design of an Asynchronous DSP Counterflow Pipeline: A Case Study in Handshake Algebra," Proc. Int'l Symp. Advanced Research in Asychronous Circuits and Systems, pp. 206-215, Nov. 1994.

[53] P.G. Lucassen and J.T. Udding, "On the Correctness of the Sproull Counterflow Pipeline Processor," Proc. Symp. Advanced Research in Asychronous Circuits and Systems, pp. 112-120, Mar. 1996.

[54] A. Yakovlev, "Designing Control Logic for Counterflow Pipeline Processor Using Petri Nets," Formal Methods in System Design, vol. 12, no. 1, pp. 39-71, Jan. 1998.

[55] M.F. Miller, K. Janik, and S.L. Lu, "Non-Stalling Counterflow Architecture," Proc. Fourth Symp. High-Performance Computer Architecture, pp. 334-341, Feb. 1998.

[56] K. Janik, SL. Lu, and M.F. Miller, "Advances of the Counterflow Pipeline Microarchitecture," proc. Int'l Symp. High-Performance Computer Architecture, pp. 230-236, Feb. 1997.

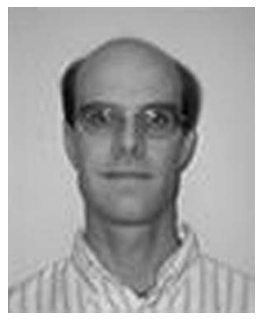

Bruce R. Childers received the BS degree (computer science, 1991) from the College of William and Mary and the PhD degree (computer science, 2000) from the University of Virginia. $\mathrm{He}$ is an assistant professor in the Department of Computer Science at the University of Pittsburgh. His research interests include computer architecture, compilers and software development tools, and embedded systems. Currently, he is researching continuous compilation, power-aware computer architecture for small and portable systems, and compiler optimization for embedded systems. He is a member of the IEEE and the IEEE Computer Society.

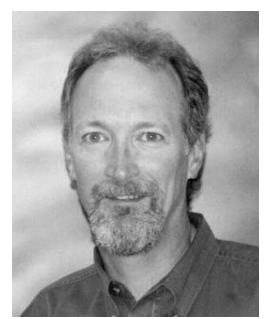

Jack W. Davidson (M'84) received the BAS and MS degrees in computer science from Southern Methodist University in 1975 and 1977, respectively. He received the $\mathrm{PhD}$ degree in computer science from the University of Arizona in 1981. He has been a member of the faculty of the University of Virginia since 1982, where he is a professor of computer science. His main research interests include compilers, code generation, optimization, embedded systems, and computer architecture. He is the author of more than 100 research articles in refereed conferences and journal articles as well as coauthor of two widely used introduction to programming textbooks. He has been a program/general chair or member of steering/program/organizing committee of many international conferences in his field and he was an associate editor of the ACM Transactions on Programming Language and Systems from 1994-2000. He is a member of the IEEE, IEEE Computer Society, ACM SIGPLAN, SIGARCH, and SIGCSE.

$\triangleright$ For more information on this or any computing topic, please visit our Digital Library at http://computer.org/publications/dlib. 